\title{
Suicidality, function and associated negative life events in an adolescent psychiatric population at 3-year follow-up
}

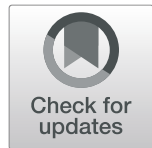

Kari Skulstad Gårdvik ${ }^{1,2^{*}}$ (D) Terje Torgersen ${ }^{3,4} \mathbb{D}$, Marite Rygg ${ }^{5,6}$ (D) Stian Lydersen ${ }^{1}\left(\mathbb{D}\right.$ and Marit Sæbø Indredavik ${ }^{5}$ (D)

\begin{abstract}
Background: We aimed to examine psychosocial function, suicidality and school dropout in a clinical psychiatric population over a 3-year period from adolescence to young adulthood and explore associations with negative life events.

Methods: This study is part of the Health Survey in Department of Children and Youth, St. Olavs hospital, Norway. In the first study visit $\left(T_{1}\right), 717$ (43.5\% of eligible) participated, aged 13-18 years (2009-2011), and 3 years later $\left(T_{2}\right)$, 570 answered a questionnaire (school functioning and negative life events), and 549 completed Kiddie SADS as telephone interview assessing DSM-IV diagnoses, psychosocial functioning and suicidality.

Results: Suicidal ideation was more frequent among girls (17.9\%) than among boys (5.4\%) (risk difference; RD = 12.5\%, $\mathrm{Cl}(7.2$ to 17.7$), p<0.001$ ), as was suicidal behavior ( $25.0 \%$ vs. $9.5 \%, \mathrm{RD}=15.5 \%, \mathrm{Cl}$ (9.2 to 21.4), $p<0.001$ ). Girls had lower psychosocial functioning than boys (Children's Global Assessment Scale; Mean score 68.2 vs. 75.2, Mean difference $=-7.0, \mathrm{Cl}(-9.4$ to -4.7$), p<0.001)$, and more school dropout $(22.5 \%$ vs. $13.2 \%, \mathrm{RD}=9.3 \%, \mathrm{Cl}(2.8$ to 15.5$), p=0.006)$. For those with a psychiatric disorder, $24.8 \%$ of girls had suicidal ideation and $30.0 \%$ suicidal behavior, which was larger than for boys ( $\mathrm{RD}=18.0 \%, \mathrm{Cl}$ (10.8 to 24.7), $p<0.001$, and $\mathrm{RD}=18.3 \%, \mathrm{Cl}$ (10.2 to 25.8), $p<0.001$, respectively). Exposure to negative life events was frequent for both genders, but more girls had experienced sexually uncomfortable or abusive situations, the last 3 years ( $23.5 \%$ vs. $2.9 \%, \mathrm{RD}=20.6 \%, \mathrm{Cl}(15.4$ to 25.7), $p<0.001$ ), and ever (44.4\% vs. $7.9 \%, \mathrm{RD}=36.5 \%, \mathrm{Cl}(29.9$ to 42.7$), p<0.001)$. Suicidal behavior was associated with having been threatened, physically harassed or violently hurt ( $\mathrm{RD}=16.7 \%, \mathrm{Cl}$ (9.5 to 23.9), $p<0.001$ ), and for girls been put into sexually uncomfortable or abusive situations $(\mathrm{RD}=20.1 \%, \mathrm{Cl}(10.4$ to 29.9$), p<0.001)$ and seen others violently hurt ( $\mathrm{RD}=14.6 \%, \mathrm{Cl}$ (3.4 to 25.8$), p=0.011$ ).
\end{abstract}

Conclusions: The high frequency of suicidality and school dropout confirms the severity of adolescent psychiatric disorders, especially among girls. Specific life events were associated risk factors and should be target points for prevention and intervention.

Keywords: Suicidal ideation, Suicidal behavior, School dropout, Adolescent, Negative life event, Longitudinal study

\footnotetext{
* Correspondence: kari.s.gardvik@ntnu.no

${ }^{1}$ Regional Centre for Child and Youth Mental Health and Child Welfare, Department of Mental Health, Faculty of Medicine and Health Sciences, Norwegian University of Science and Technology, Trondheim, Norway

${ }^{2}$ Department of Children and Youth, Division of Mental Health Care, St. Olavs Hospital, Trondheim University Hospital, Trondheim, Norway

Full list of author information is available at the end of the article
} changes were made. The images or other third party material in this article are included in the article's Creative Commons licence, unless indicated otherwise in a credit line to the material. If material is not included in the article's Creative Commons licence and your intended use is not permitted by statutory regulation or exceeds the permitted use, you will need to obtain permission directly from the copyright holder. To view a copy of this licence, visit http://creativecommons.org/licenses/by/4.0/ The Creative Commons Public Domain Dedication waiver (http://creativecommons.org/publicdomain/zero/1.0/) applies to the data made available in this article, unless otherwise stated in a credit line to the data. 


\section{Background}

Adolescence is the period for transitioning into young adulthood and is usually a time of life characterized by good physical health [1]. However, the majority of mental disorders develop during adolescence and contribute to reduced psychosocial function [1-3]. Suicidal symptoms increase during this developmental period [4-6], with a rapid shift from suicidal ideation to suicidal behavior $[5,7,8]$, and an estimated lifetime prevalence of suicidal ideation and suicide attempts of $12.1-33 \%$ and $4.1-9.3 \%$, respectively $[5,9]$. The prevalence of selfharm, defined broadly regardless of motivation and intention to die, has increased among Norwegian adolescents from 4.1 to $16.2 \%$ between 2002 and 2018 [10]. Second to road injury, suicide is the most common cause of death among young people worldwide, uncommon before 15 years of age but the frequency increases through adolescence [6,11-13]. The prevalence across all ages, countries and gender is $3.77 / 100000$, and in Norway 3.00/100000 [12]. Suicide characteristics differ by gender [6, 12-15], with girls having higher rates of suicidal thoughts and behavior, and boys highest rates of committed suicide. Suicidal ideation and behavior are common in patients with psychiatric disorders $[5,6,16]$ and are more than three times more frequent in clinical samples of youth than in the general population [7, 17]. Accordingly, the rise of suicidal thoughts and behavior through adolescence coexists with increasing frequencies of psychiatric disorders and related psychopathology that by itself provide higher suicide risk, as for example depression, substance use and some anxiety disorders $[5,7,16]$.

It is common to have experienced negative or stressful life events or adversities from childhood to young adulthood $[18,19]$. Many different life events are found to be associated with youth suicidal symptoms [20-22]. Such events may include being exposed or witnessed to violence, sexual trauma, or other injury and trauma [19], which are more frequent in clinical psychiatric samples than in the general population $[23,24]$. In a systematic review, young people with attempted suicide were more likely to have experienced stressful life events than those with suicidal ideation [20]. A meta-analysis provided strong evidence that early exposure to any interpersonal violence increased the risk of suicide attempts [25]. Many other negative life events have shown associations to suicidal ideation, behavior or committed suicide, as for example death of a parent or a loved one [26, 27], experiences of disasters or accidents [27], peer victimization [28] and multiple other family factors [29, 30]. Experiencing negative life events during demanding developmental periods in childhood and adolescence may increase vulnerability to mental distress by inducing biological changes with long-term effects on nervous, endocrine and immune systems $[20,31]$. Thus, negative life events may increase the risk for psychiatric symptoms, including suicidal behavior in vulnerable individuals [20, 32].

Psychiatric disorders and comorbidities in early years influence academic functioning, and may subsequently lead to increased risk of dropping out of school [33] and receiving unemployment benefits or social insurance support [33]. In a population-based study in Central Norway, $17 \%$ was registered as being high school dropouts at age 24 [33], and more boys than girls were found to be non-completers in another Norwegian populationbased survey [34]. According to World Health Organization, education and health are strongly linked [35]. School dropout was associated with poor mental health in a Danish population-based study [36], and school dropout involve heavy and enduring individual and social costs [37]. The link between suicidal symptoms, psychosocial and school function seems to be bidirectional; Adolescent self-harm or suicidal behavior are found to be associated with later mental health disorders and worse long-term functioning in young adulthood [38, 39]. According to a systematic review with meta-analysis of longitudinal studies, adolescents and young adults with school failure were at higher risk of suicide attempts [40]. There are many risk factors for school dropout [41], and reasons for leaving school vary widely [42]. Negative or stressful life events are found to be associated with intentions of and actual dropout [43], including conflicts with authorities for boys, and relational problems for girls [44]. High school students exposed to severe acute stressors are immediately vulnerable to dropping out [37].

The objective of the present study was to examine suicidality and functioning 3 years after referral to Child and Adolescent Mental Health Services. We aimed to assess psychosocial function, suicidal ideation, suicidal behavior, and school dropout, in the total sample and specified by psychiatric disorders, and furthermore to investigate associations with negative life events. We set out to specify analyses for girls and boys and explore gender differences. Hypotheses were that present suicidal symptoms and school dropout were associated with co-occurring exposure to negative life events, and furthermore, that frequencies differed between girls and boys, with girls having higher rates of suicidal symptoms and boys more school dropout.

\section{Method}

\section{Study design}

The Health Survey in Department of Children and Youth, Division of Mental Health Care, St. Olavs hospital, Trondheim University Hospital, Norway (St. Olav CAP Survey), is a prospective longitudinal cohort study of a defined clinical population assessed at two time 
points. Design and procedures are thoroughly described in former publications $[45,46]$. At time point $1\left(\mathrm{~T}_{1}\right)$ (2009-2011), all patients aged 13-18 years who visited the Department of Children and Youth at least once over a 2-year study period, received oral and written invitations at their first attendance. The exclusion criteria were difficulties in answering the survey due to low cognitive function, visual impairments, insufficient language skills, or an unstable psychiatric state. Emergency patients were invited to take part once they entered a stable phase. The participants and their parents received standard application of services. They gave written informed consent to extract diagnostic data from clinical charts and respond to an electronic survey. At 3-year study follow-up $\left(\mathrm{T}_{2}\right)$ (2012-2014), age 16-21 years, data were collected from the $T_{1}$ enrolled sample and their parents, by an electronic survey and a diagnostic telephone interview performed by trained professionals.

\section{Participants}

In the $\mathrm{T}_{1}$ study period, 2032 adolescent patients had at least one attendance in the Department of Children and Youth $[45,46]$. Figure 1 shows the participant flow in each stage of the survey. At $\mathrm{T}_{1}, n=717$ participated (393 (54.8\%) girls). At $\mathrm{T}_{2}$, all $\mathrm{T}_{1}$ participants who previously consented to further inquiry were invited (eligible $n=$ 685), of whom 570 (83\% of eligible) completed the follow-up questionnaire (324 (56.8\%) girls), and 549 (80\%) completed the diagnostic interview (308 (56.1\%) girls).

\section{Participants vs. non-participants}

To explore the representativeness of the study population at $\mathrm{T}_{1}$, anonymous information about the total clinical population was collected from annual reports from the Department of Children and Youth, 2009-2011, as previously published $[45,46]$. All adolescents in the

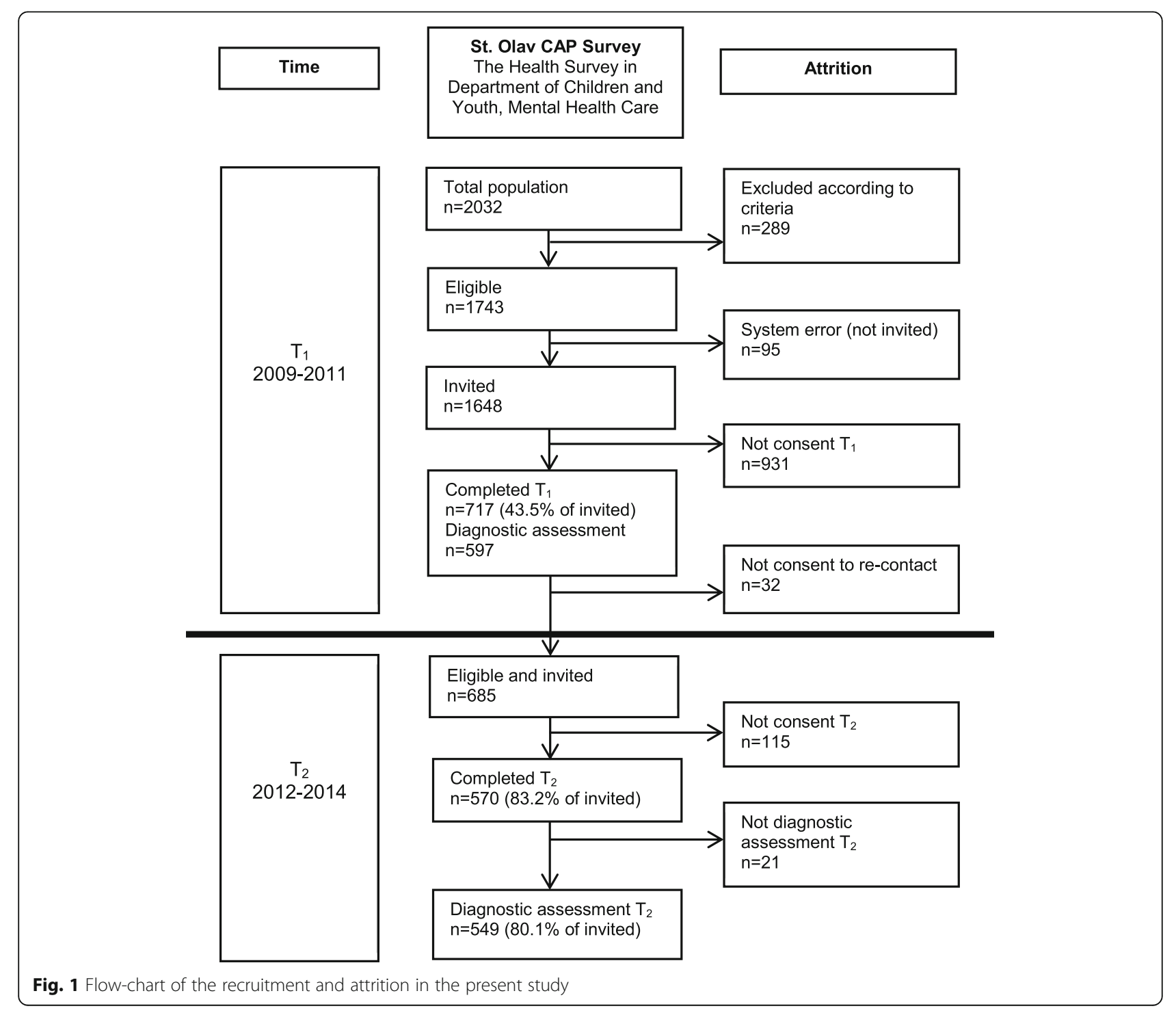


study period $(n=2032)$ minus those excluded $(n=289)$ were defined as reference population $(n=1743)$. The main reason for referral, age and gender were similar between participants $(n=717,41.1 \%)$ and non-participants $(n=1026,58.9 \%)$ (data not shown). Participants were 0.27 years older: Mean (SD) 15.7 (1.7) vs. 15.4 (2.0), and there were more girls among the participants: 393 $(54.8 \%)$ vs. 509 (49.6\%). Among those with participation at $\mathrm{T}_{1}$, there were 570 participants and 147 nonparticipants at $T_{2}$. In depth attrition analyses are reported in a former publication [46]. Age and socioeconomic status were similar among participants and nonparticipants.

\section{Measures}

Psychiatric Diagnoses at $T_{2}$ were set using the semistructured Schedule for Affective Disorders and Schizophrenia for School-Age Children (K-SADS) [47] according to the Diagnostic and Statistical Manual of Mental Disorders IV Text revision (DSM-IV-TR) [48]. Psychometric properties of the K-SADS, including reliability and validity, are found to be excellent [47], and the interview has previously been applied to populations in young adulthood $[49,50]$. Adolescents were interviewed by telephone by trained interviewers, all with graduate degree in medicine or psychology and experience in child and adolescent psychiatric assessment. The interviewers met regularly with a supervisor, an experienced child and adolescent psychiatrist, to assure the quality and harmonization of the diagnostic assessment. All were blinded to $T_{1}$ diagnoses. Inter-rater reliability in terms of negative agreement and positive agreement as recommended by van de Vet et al. [51], was assessed using second ratings for 28 of the taped telephone interviews. Positive agreement varied from 0.615 to 1.000 , and negative agreement varied from 0.884 to 1.000 [46]. The underlying contingency tables showing agreement are previously reported [46].

In the present study, disorders were grouped into the following categories, based on DSM-IV diagnoses at $\mathrm{T}_{2}$; Any psychiatric disorder, Anxiety disorders (DSM-codes 300, 308, 309), Mood disorders (DSM-codes 296, 300.4, 311), ADHD (DSM-code 314) and Other (DSM-codes 291, 292, 295, 298, 299, 301, 303, 304, 305, 307, 312, $313,316)$. Due to few participants in some diagnostic groups, for example autism and eating disorders, and especially when examining suicidality and school dropout, we chose to merge children with these diagnoses into "other psychiatric disorders" for the purpose of this manuscript.

The Children's Global Assessment Scale (CGAS) [52] was used to rate general psychosocial functioning on a scale from 1 (extremely impaired, needs constant supervision) to 100 (superior functioning), based on K-
SADS interview. The CGAS is designed for children under 18 years, but was in this study used for all participants, also those above the age of 18 years. The interrater reliability for CGAS in terms of intraclass correlation coefficient (ICC) was 0.835 , based on second ratings for 28 of the taped telephone interviews. Details are given in the Supplementary material (Table S1).

Suicidal ideation or behavior were measured at $T_{2}$ by asking the following questions during K-SADS interview:

Suicidal ideation; "Sometimes children who get upset or feel bad think about dying or even killing themselves. Have you ever had such thoughts? How would you do it? Did you have a plan?" Assessed and scored as; 0; No information, 1; Not at all, 2; Infrequent or vague thoughts of suicide (e.g., less than once per month), or 3; Recurrent thoughts of suicide. As measure of Suicidal ideation, we used "infrequent or vague thoughts" (2) or "recurrent thoughts of suicide" (3), presently at $\mathrm{T}_{2}$.

Suicidal acts or attempts; "Have you actually tried to kill yourself? When? What did you do? Any other things? Did you really want to die? How close did you come to doing it? Was anybody in the room? In the apartment? Did you tell them in advance? How were you found? Did you really want to die? Did you ask for any help after you did it?" Assessed and scored as; 0; No information, 1; No attempt, 2; Preparations with no actual intent to die (e.g., held pills in hand) or planned attempt but did not follow through, 3; Self injurious behavior with any suicidal intent. There was one more assessment; "Ever attempted suicide", scored as yes or no. In the present study, Suicidal behavior included "preparations or planned attempt" (2) or "self injurious behavior with any suicidal intent" (3), presently at $\mathrm{T}_{2}$, or yes to the question: "Ever attempted suicide".

School dropout was self-reported at $\mathrm{T}_{2}$ based on answer "yes" to the following question: "Have you canceled your education (dropped out)?"

Negative life events were registered by self-report at both $\mathrm{T}_{1}$ and $\mathrm{T}_{2}$. At $\mathrm{T}_{1}$, the following questions were asked: "Have any of the following things happened to you?"; "That someone in your family has been seriously ill", "Death of a loved one", "A catastrophe (fire, avalanche, tidal wave, hurricane, etc.)", "A serious accident (ex: a very serious car accident)", "Been violently hurt (beaten or injured)", "Seen others violently hurt", "Been put in sexually uncomfortable/abusive situations by someone about your age", "Been put in sexually uncomfortable/abusive situations by an adult", "Been threatened or physically harassed by other students at school for a long time", "Received painful or frightening treatment at the hospital while being treated for an illness or injury". These items were also used in the YoungHUNT3 study (https://www.ntnu.edu/hunt/data/que). 
At $T_{2}$, the same questions were asked, and with a supplementary question: "Been seriously ill or injured".

The answering opportunities were at $\mathrm{T}_{1}$; "No", "Yes, last year" and "Yes, in my life", and at $\mathrm{T}_{2}$; "No", "Yes, last year" and "Yes, last three years". In the present study, negative life events defined as "last 3 years" were events measured at $\mathrm{T}_{2}$ only, and negative life events defined as "ever" were measured at $\mathrm{T}_{1}$ or $\mathrm{T}_{2}$.

Socioeconomic Status (SES) was measured at $\mathrm{T}_{1}$ by the highest level of mothers' education, divided into eight categories: 1) less than 9-year primary school; 2) completed 9-year primary school; 3) one or two years in high school; 4) completed high school; 5) completed high school and one-year education/training after high school; 6) academy/university for up to and including 4 years; 7) academy/university for 5 years or more; 8) academy/university including $\mathrm{PhD}$.

\section{Statistical analyses}

We compared proportions using the Newcombe hybrid score confidence intervals, as recommended by Fagerland, Lydersen and Laake [53], and the Pearson Chi squared test. Confidence intervals and tests for differences in psychosocial functioning between girls and boys were based on Student's t-test for independent samples. We used binary linear regression with suicidal ideation, suicidal behavior or school dropout at $\mathrm{T}_{2}$ as dependent variables and negative life events reported at $T_{1}$ and $T_{2}$ as covariates, one at a time, to study their associations. The coefficients in binary linear regression represent risk differences. These regression analyses were carried out unadjusted and adjusted for SES as a possible confounder where relevant. Some estimates including suicidal behavior could not be computed when adjusting for SES due to non-convergence of the calculations. We report 95\% confidence intervals $(\mathrm{CI})$ where relevant, and two-sided $p$ values $<0.05$ were considered statistically significant. Binary linear regression and the Newcombe CI were performed in Stata 16, and the other in SPSS 25.

\section{Results}

\section{Suicidal measures and functioning}

At $\mathrm{T}_{2}$, psychosocial functioning CGAS score was mean 71.3 (standard deviation 14.5) (Table 1). Girls had lower CGAS score than boys (mean 68.2 vs. 75.2, mean difference $=-7.0$, CI $(-9.4$ to -4.7$), p<0.001)$. The frequency of suicidal ideation was $12.4 \%$, girls $17.9 \%$ and boys $5.4 \%$ (risk difference; $\mathrm{RD}=12.5 \%$, CI (7.2 to 17.7), $p<0.001$ ) (Table 1). Similar gender differences were found in suicidal behavior, were girls had the highest frequencies of suicidal attempts ever $(25.0 \%$ vs. $9.5 \%, \mathrm{RD}=15.5 \%$, CI (9.2 to 21.4), $p<0.001)$. School dropout was more frequent for girls than boys ( $22.5 \%$ vs. $13.2 \%$, RD $=9.3 \%$, CI (2.8 to 15.5$), p=0.006$ ). Among those with a psychiatric disorder, suicidal ideation was higher among girls (24.8\%), and suicidal behavior even higher (30.0\%), RD for gender differences $18.0 \%$, CI (10.8 to 24.7), $p<0.001$, and $18.3 \%, \mathrm{CI}$ (10.2 to 25.8), $p<0.001$, respectively (Table 2). Specified by psychiatric disorder, girls had lower CGAS and higher frequencies of suicidal measures than boys in all diagnostic groups. The frequencies of suicidal ideation and behavior were highest in mood

Table 1 Clinical characteristics, psychosocial functioning, suicidal measures and school dropout at 3-year follow up

\begin{tabular}{|c|c|c|c|c|c|c|c|c|c|c|c|c|}
\hline \multirow[b]{2}{*}{ Follow-up $\left(\mathrm{T}_{2}\right)$} & \multicolumn{3}{|c|}{ Total } & \multicolumn{3}{|c|}{ Girls } & \multicolumn{3}{|c|}{ Boys } & \multicolumn{3}{|l|}{ Girls versus Boys } \\
\hline & $\mathbf{n}$ & Mean & (SD) & $\mathbf{n}$ & Mean & (SD) & $\mathbf{n}$ & Mean & (SD) & Mean difference & $95 \% \mathrm{Cl}^{\mathrm{a}}$ & $\begin{array}{l}p- \\
\text { value }^{a}\end{array}$ \\
\hline Age (years) & 570 & 18.7 & $(1.7)$ & 324 & 19.0 & $(1.7)$ & 246 & 18.3 & $(1.6)$ & 0.7 & $(0.4$ to 0.9$)$ & $<0.001$ \\
\hline SES & 404 & 4.8 & $(1.7)$ & 221 & 4.9 & $(1.7)$ & 183 & 4.8 & $(1.8)$ & 0.1 & $(-0.3$ to 0.4$)$ & 0.714 \\
\hline \multirow[t]{2}{*}{ CGAS } & 549 & 71.3 & $(14.5)$ & 308 & 68.2 & $(15.5)$ & 241 & 75.2 & $(12.0)$ & -7.0 & $\begin{array}{l}(-9.4 \text { to } \\
-4.7)\end{array}$ & $<0.001$ \\
\hline & & Proportion & (\%) & & Proportion & (\%) & & Proportion & (\%) & $\begin{array}{l}\text { Risk difference } \\
(\%)\end{array}$ & $95 \% \mathrm{Cl}^{\mathrm{b}}$ & $\begin{array}{l}p- \\
\text { value }^{c}\end{array}$ \\
\hline Suicidal ideation $^{d}$ & 549 & $68 / 548$ & $(12.4)$ & 308 & $55 / 307$ & $(17.9)$ & 241 & $13 / 241$ & $(5.4)$ & 12.5 & (7.2 to 17.7$)$ & $<0.001$ \\
\hline Suicidal behavior & 549 & $100 / 549$ & $(18.2)$ & 308 & $77 / 308$ & $(25.0)$ & 241 & $23 / 241$ & $(9.5)$ & 15.5 & (9.2 to 21.4$)$ & $<0.001$ \\
\hline $\begin{array}{l}\text { - Suicidal attempts } \\
\text { presently }\end{array}$ & 549 & $9 / 549$ & $(1.6)$ & 308 & $9 / 308$ & $(2.9)$ & 241 & $0 / 241$ & $(0.0)$ & 2.9 & (0.8 to 5.5$)$ & 0.007 \\
\hline - Suicidal attempts ever & 549 & $100 / 549$ & $(18.2)$ & 308 & $77 / 308$ & $(25.0)$ & 241 & $23 / 241$ & $(9.5)$ & 15.5 & (9.2 to 21.4$)$ & $<0.001$ \\
\hline School dropout ${ }^{e}$ & 570 & $101 / 546$ & $(18.5)$ & 324 & $70^{f} / 311$ & $(22.5)$ & 246 & $31 / 235$ & $(13.2)$ & 9.3 & $(2.8$ to 15.5$)$ & 0.006 \\
\hline
\end{tabular}

Note: SES Socioeconomic status, SD Standard Deviation, CGAS Children Global Assessment Scale (psychosocial functioning) $(1-100,1=$ worst, 100 = best)

${ }^{a}$ Confidence intervals and tests for differences between girls and boys were based on Student's t-test for independent samples

${ }^{b}$ Newcombe hybrid score

c Pearson Chi squared test

${ }^{d}$ Suicidal ideation is defined as suicidal thoughts occasionally or often

e School dropout includes patients answering yes to the question "Have you canceled your education (dropped out)?"

${ }^{f}$ Of these, 6 had given childbirth 
Table 2 General psychosocial functioning, suicidal ideation or behavior and school dropout at 3-year follow up, specified by psychiatric disorders

\begin{tabular}{|c|c|c|c|c|c|c|c|c|c|}
\hline \multirow{2}{*}{ Any psychiatric disorder $^{\mathrm{a}}$} & \multicolumn{2}{|c|}{ Total $(n=549)$} & \multicolumn{2}{|c|}{ Girls $(n=308)$} & \multicolumn{2}{|c|}{ Boys $(n=241)$} & \multicolumn{3}{|l|}{ Girls versus Boys } \\
\hline & \multicolumn{2}{|l|}{$n=385$} & \multicolumn{2}{|l|}{$n=223$} & \multicolumn{2}{|l|}{$n=162$} & \multirow[b]{2}{*}{ Mean difference } & \multirow[b]{2}{*}{$95 \% \mathrm{Cl}^{\mathrm{d}}$} & \multirow[b]{2}{*}{$p$-value ${ }^{d}$} \\
\hline & Mean & (SD) & Mean & (SD) & Mean & (SD) & & & \\
\hline \multirow[t]{2}{*}{ CGAS } & 66.8 & $(14.0)$ & 63.3 & $(14.4)$ & 71.6 & (12.0) & -8.3 & $(-10.9$ to -5.6$)$ & $<0.001$ \\
\hline & $\mathbf{n}$ & (\%) & $\mathbf{n}$ & (\%) & $\mathbf{n}$ & (\%) & RD (\%) & $95 \% \mathrm{Cl}$ of $\mathrm{RD}^{\mathrm{e}}$ & $p$-value ${ }^{f}$ \\
\hline Suicidal ideation ${ }^{\mathrm{b}}$ & $66 / 384$ & $(17.2)$ & $55 / 222$ & (24.8) & $11 / 162$ & (6.8) & 18.0 & (10.8 to 24.7$)$ & $<0.001$ \\
\hline Suicidal behavior ${ }^{c}$ & $86 / 385$ & $(22.3)$ & $67 / 223$ & $(30.0)$ & $19 / 162$ & $(11.7)$ & 18.3 & (10.2 to 25.8$)$ & $<0.001$ \\
\hline School dropout & $77 / 361$ & $(21.3)$ & $53 / 211$ & (25.1) & $24 / 150$ & (16.0) & 9.1 & (0.5 to 17.1$)$ & 0.037 \\
\hline \multirow[t]{2}{*}{ Anxiety disorders } & $n=218$ & & $n=168$ & & $n=50$ & & & & \\
\hline & Mean & (SD) & Mean & (SD) & Mean & (SD) & Mean difference & $95 \% \mathrm{Cl}$ of Difference & $p$-value \\
\hline \multirow[t]{2}{*}{ CGAS } & 61.6 & $(13.3)$ & 60.5 & (13.9) & 65.2 & (10.4) & -4.7 & $(-8.2$ to -1.0$)$ & 0.012 \\
\hline & $\mathrm{n}$ & (\%) & $\mathbf{n}$ & (\%) & $\mathbf{n}$ & (\%) & RD (\%) & $95 \% \mathrm{Cl}$ of $\mathrm{RD}$ & $p$-value \\
\hline Suicidal ideation & $48 / 218$ & $(22.0)$ & $43 / 168$ & (25.6) & $5 / 50$ & (10.0) & 15.6 & (2.7 to 24.7 ) & 0.019 \\
\hline Suicidal behavior & $64 / 218$ & $(29.4)$ & $55 / 168$ & (32.7) & $9 / 50$ & (18.0) & 14.7 & (0.3 to 25.8$)$ & 0.045 \\
\hline School dropout & $50 / 205$ & $(24.4)$ & $42 / 159$ & (26.4) & $8 / 46$ & (17.4) & 9.0 & $(-5.7$ to 20.1$)$ & 0.209 \\
\hline \multirow[t]{2}{*}{ Mood disorders } & $n=98$ & & $n=80$ & & $n=18$ & & & & \\
\hline & Mean & (SD) & Mean & (SD) & Mean & (SD) & Mean difference & $95 \% \mathrm{Cl}$ of Difference & $p$-value \\
\hline \multirow[t]{2}{*}{ CGAS } & 55.8 & $(11.3)$ & 54.5 & (11.4) & 61.4 & (9.6) & -6.9 & $(-12.7$ to -1.2$)$ & 0.018 \\
\hline & $\mathrm{n}$ & (\%) & $\mathbf{n}$ & (\%) & $\mathbf{n}$ & (\%) & RD (\%) & $95 \% \mathrm{Cl}$ of $\mathrm{RD}$ & $p$-value \\
\hline Suicidal ideation & $42 / 98$ & $(42.9)$ & $38 / 80$ & $(47.5)$ & $4 / 18$ & (22.2) & 25.3 & $(-0.0$ to 42.4$)$ & 0.050 \\
\hline Suicidal behavior & $39 / 98$ & $(39.8)$ & $36 / 80$ & $(45.0)$ & $3 / 18$ & (16.7) & 28.3 & (3.5 to 43.7 ) & 0.026 \\
\hline School dropout & $30 / 90$ & $(33.3)$ & $23 / 73$ & (31.5) & $7 / 17$ & $(41.2)$ & -9.7 & $(-34.4$ to 13.0$)$ & 0.446 \\
\hline \multirow[t]{2}{*}{ ADHD } & $n=211$ & & $n=99$ & & $n=112$ & & & & \\
\hline & Mean & (SD) & Mean & (SD) & Mean & (SD) & Mean difference & $95 \% \mathrm{Cl}$ of Difference & $p$-value \\
\hline \multirow[t]{2}{*}{ CGAS } & 69.2 & $(13.6)$ & 65.9 & $(14.9)$ & 72.1 & (11.6) & -6.2 & $(-9.9$ to -2.6$)$ & 0.001 \\
\hline & n & (\%) & $\mathbf{n}$ & (\%) & $\mathbf{n}$ & (\%) & RD (\%) & $95 \% \mathrm{Cl}$ of $\mathrm{RD}$ & $p$-value \\
\hline Suicidal ideation & $22 / 210$ & $(10.5)$ & $16 / 98$ & $(16.3)$ & $6 / 112$ & (5.4) & 10.9 & (2.6 to 20.0$)$ & 0.010 \\
\hline Suicidal behavior & $41 / 211$ & $(19.4)$ & $27 / 99$ & $(27.3)$ & $14 / 112$ & $(12.5)$ & 14.8 & (4.0 to 25.5$)$ & 0.007 \\
\hline School dropout & $37 / 197$ & $(18.8)$ & $23 / 93$ & $(24.7)$ & $14 / 104$ & (13.5) & 11.3 & (0.3 to 22.3$)$ & 0.043 \\
\hline \multirow[t]{2}{*}{ Other psychiatric disorders } & $n=120$ & & $n=59$ & & $n=61$ & & & & \\
\hline & Mean & (SD) & Mean & (SD) & Mean & (SD) & Mean difference & $95 \% \mathrm{Cl}$ of Difference & $p$-value \\
\hline \multirow[t]{2}{*}{ CGAS } & 63.2 & $(14.5)$ & 57.7 & $(14.2)$ & 68.4 & (12.9) & -10.7 & $(-15.7$ to -5.8$)$ & $<0.001$ \\
\hline & $\mathrm{n}$ & (\%) & $\mathbf{n}$ & (\%) & $\mathbf{n}$ & (\%) & RD (\%) & $95 \% \mathrm{Cl}$ of $\mathrm{RD}$ & $p$-value \\
\hline Suicidal ideation & $27 / 120$ & $(22.5)$ & $20 / 59$ & (33.9) & $7 / 61$ & (11.5) & 22.4 & (7.5 to 36.4 ) & 0.003 \\
\hline Suicidal behavior & $31 / 120$ & $(25.8)$ & $23 / 59$ & $(39.0)$ & $8 / 61$ & (13.1) & 25.9 & (10.2 to 40.1$)$ & 0.001 \\
\hline School dropout & $31 / 114$ & $(27.2)$ & 19/57 & (33.3) & $12 / 57$ & $(21.0)$ & 12.3 & $(-4.1$ to 27.8$)$ & 0.141 \\
\hline
\end{tabular}

Note: SD Standard Deviation, CGAS Children Global Assessment Scale (general psychosocial functioning) $(1-100,1=$ worst, $100=$ best), RD Risk difference

${ }^{a}$ Psychiatric disorder includes both primary and additional diagnoses

${ }^{b}$ Suicidal ideation is defined as suicidal thoughts occasionally or often

c Suicidal behavior is defined as suicidal acts or attempts, presently at $T_{2}$ or ever, also suicidal acts and attempts with suicidal thoughts

${ }^{d}$ Confidence intervals and tests for differences between girls and boys were based on Student's t-test for independent samples

e Newcombe hybrid score

${ }^{f}$ Pearson Chi squared test

disorders and lowest in ADHD. For school dropout, gender difference was only found among patients with ADHD, with highest frequencies among girls (24.7\% vs. $13.5 \%, \mathrm{RD}=11.3 \%$, CI (0.3 to 22.3), $p=0.043$ ).
Negative life events

Having serious illness of someone in family or death of a loved one, were the most common negative life events in this study (57.7\% last 3 years and $85.7 \%$ ever), with 
higher frequencies among girls than boys only for the last 3 years $(63.2 \%$ vs. $50.4 \%, \mathrm{RD}=12.8 \%, \mathrm{CI}$ ( 4.5 to $20.8), p=0.002$ ) (Table 3$)$. Having been seriously ill, injured or received painful or frightening treatment in hospital were more frequent among girls than boys both for the last 3 years and ever $(26.5 \%$ vs. $16.5 \%, \mathrm{RD}=10.0 \%$, CI (3.1 to 16.6 ), $p=0.005$, and $38.0 \%$ vs. $27.0 \%, \mathrm{RD}=$ $11.0 \%$, CI (7.6 to 22.5), $p<0.001$, respectively). Ever been exposed to a serious accident or catastrophe, were more frequent among girls $(37.0 \%$ vs. $24.0 \%, \mathrm{RD}=13.0 \%$, CI (5.4 to 20.4), $p=0.001$ ). There were highly significant differences between girls and boys in having been put into sexually uncomfortable or abusive situations, both during the last 3 years and ever $(23.5 \%$ vs. $2.9 \%, \mathrm{RD}=$ $20.6 \%$, CI (15.4 to 25.7 ), $p<0.001$, and $44.4 \%$ vs. $7.9 \%$, $\mathrm{RD}=36.5 \%$, CI (29.9 to 42.7$), p<0.001$, respectively).

\section{Associations}

Binary linear regression with suicidal ideation as dependent variable and negative life events as covariates showed associations for several life events (Table 4). After adjustment for SES, the strongest associations were for been threatened, physically harassed or violently hurt $(\mathrm{RD}=8.9 \%, \mathrm{CI}$ (2.0 to 15.9$), p=0.012)$ and having been put into sexually uncomfortable or abusive situations ( $\mathrm{RD}=10.4 \%$, CI (1.8 to 19.0$), p=0.018)$. Gender-specific analyses adjusted for SES, showed associations with having serious illness of someone in the family or death of a loved one, and being threatened, physically harassed or violently hurt for girls, but no associations were present for boys.

With suicidal behavior as dependent variable, adjusted associations were present for been seriously ill or injured ( $\mathrm{RD}=10.6 \%, \mathrm{CI}$ (2.8 to 18.4), $p=0.008$ ), exposure to a serious accident or catastrophe ( $\mathrm{RD}=10.1 \%$, CI (1.9 to 18.3), $p=0.015)$, and been threatened, physically harassed or violently hurt ( $\mathrm{RD}=16.7 \%$, CI (9.5 to 23.9 ), $p<$ 0.001) (Table 5). Having seen others violently hurt was associated with suicidal behavior in girls only $(\mathrm{RD}=$ $14.6 \%$, CI (3.4 to 25.8), $p=0.011$ ). Some estimates could not be adjusted for SES due to non-convergence of the calculations. Thus, the association with having been put into sexually uncomfortable or abusive situations $(\mathrm{RD}=$ $21.8 \%$, CI (13.6 to 29.9), $p<0.001$ ) could not be adjusted for SES, neither could the corresponding association that was present only for girls ( $\mathrm{RD}=20.1 \%$, CI (10.4 to 29.9 ), $p<0.001)$. Having been threatened, physically harassed or violently hurt was related to suicidal behavior for both girls ( $\mathrm{RD}=17.6 \%$, CI (6.9 to 28.3), $p=0.001$ ) and unadjusted for boys ( $\mathrm{RD}=12.0 \%$, CI (3.8 to 20.2), $p=$ $0.004)$. There was an association between SES and suicidal behavior ( $\mathrm{RD}=-2.3 \%$, CI $(-4.4$ to -0.8$), p=$ $0.005)$. Specified by diagnostic groups, associations with suicidal behavior were highly significant for Mood

Table 3 Negative life events at 3-year follow up

\begin{tabular}{|c|c|c|c|c|c|c|c|c|c|c|c|c|c|c|c|c|}
\hline \multirow{3}{*}{$\begin{array}{l}\text { Self-reported questionnaire } \\
\left(T_{1} \text { and } T_{2}\right)^{a}\end{array}$} & \multicolumn{4}{|c|}{ Total $(n=570)$} & \multicolumn{4}{|c|}{ Girls $(n=324)$} & \multicolumn{4}{|c|}{ Boys $(n=246)$} & \multicolumn{4}{|c|}{ Girls versus Boys } \\
\hline & \multicolumn{2}{|c|}{$\begin{array}{l}\text { Last } 3 \\
\text { years }^{\text {b }}\end{array}$} & \multicolumn{2}{|c|}{ Ever $^{\mathbf{b}}$} & \multicolumn{2}{|c|}{$\begin{array}{l}\text { Last } 3 \\
\text { years }\end{array}$} & \multicolumn{2}{|l|}{ Ever } & \multicolumn{2}{|c|}{$\begin{array}{l}\text { Last } 3 \\
\text { years }\end{array}$} & \multicolumn{2}{|l|}{ Ever } & \multicolumn{2}{|c|}{ Last 3 years } & \multicolumn{2}{|l|}{ Ever } \\
\hline & $\mathrm{n}$ & $(\%)$ & $\mathrm{n}$ & (\%) & $\mathrm{n}$ & $(\%)$ & $\mathrm{n}$ & (\%) & $\mathrm{n}$ & $(\%)$ & $\mathrm{n}$ & $(\%)$ & $\begin{array}{l}95 \% \\
\mathrm{Cl}^{\mathrm{c}}\end{array}$ & $p^{d}$ & $\begin{array}{l}95 \% \\
\mathrm{Cl}\end{array}$ & $p$ \\
\hline $\begin{array}{l}\text { Serious illness of someone in family or } \\
\text { death of a loved one }\end{array}$ & $\begin{array}{l}326 / \\
565\end{array}$ & $(57.7)$ & $\begin{array}{l}485 / \\
566\end{array}$ & $(85.7)$ & $\begin{array}{l}204 / \\
323\end{array}$ & $(63.2)$ & $\begin{array}{l}284 / \\
323\end{array}$ & $(87.9)$ & $\begin{array}{l}122 / \\
242\end{array}$ & $(50.4)$ & $\begin{array}{l}201 / \\
242\end{array}$ & $(83.1)$ & $\begin{array}{l}(4.5 \text { to } \\
20.8)\end{array}$ & 0.002 & $\begin{array}{l}(-0.9 \\
\text { to } \\
11.0)\end{array}$ & 0.080 \\
\hline $\begin{array}{l}\text { Been seriously ill or injured, received } \\
\text { painful or frightening treatment at } \\
\text { hospital }\end{array}$ & $\begin{array}{l}126 / \\
566\end{array}$ & $(22.3)$ & $\begin{array}{l}178 / \\
566\end{array}$ & (31.4) & $\begin{array}{l}86 / \\
324\end{array}$ & $(26.5)$ & $\begin{array}{l}123 / \\
324\end{array}$ & (38.0) & $\begin{array}{l}40 / \\
242\end{array}$ & $(16.5)$ & $\begin{array}{l}55 / \\
242\end{array}$ & (22.7) & $\begin{array}{l}\text { (3.1 to } \\
16.6)\end{array}$ & 0.005 & $\begin{array}{l}(7.6 \\
\text { to } \\
22.5)\end{array}$ & $\begin{array}{l}< \\
0.001\end{array}$ \\
\hline $\begin{array}{l}\text { Exposed to a serious accident or } \\
\text { catastrophe }\end{array}$ & $\begin{array}{l}74 / \\
566\end{array}$ & $(13.1)$ & $\begin{array}{l}178 / \\
566\end{array}$ & (31.4) & $\begin{array}{l}45 / \\
324\end{array}$ & $(13.9)$ & $\begin{array}{l}120 / \\
324\end{array}$ & $(37.0)$ & $\begin{array}{l}29 / \\
242\end{array}$ & $(12.0)$ & $\begin{array}{l}58 / \\
242\end{array}$ & $(24.0)$ & $\begin{array}{l}(-3.9 \\
\text { to } 7.4)\end{array}$ & 0.506 & $\begin{array}{l}(5.4 \\
\text { to } \\
20.4)\end{array}$ & 0.001 \\
\hline $\begin{array}{l}\text { Been threatened, physically harassed } \\
\text { or violently hurt }\end{array}$ & $\begin{array}{l}124 / \\
566\end{array}$ & $(21.9)$ & $\begin{array}{l}262 / \\
566\end{array}$ & $(46.3)$ & $\begin{array}{l}77 / \\
324\end{array}$ & $(23.8)$ & $\begin{array}{l}160 / \\
324\end{array}$ & (49.4) & $\begin{array}{l}47 / \\
242\end{array}$ & $(19.4)$ & $\begin{array}{l}101 / \\
242\end{array}$ & $(41.7)$ & $\begin{array}{l}(-2.6 \\
\text { to } \\
11.0)\end{array}$ & 0.216 & $\begin{array}{l}(-0.6 \\
\text { to } \\
15.8)\end{array}$ & 0.088 \\
\hline Seen others violently hurt & $\begin{array}{l}131 / \\
566\end{array}$ & $(23.1)$ & $\begin{array}{l}241 / \\
566\end{array}$ & (42.6) & $\begin{array}{l}65 / \\
324\end{array}$ & $(20.1)$ & $\begin{array}{l}140 / \\
324\end{array}$ & $(43.2)$ & $\begin{array}{l}66 / \\
242\end{array}$ & $(27.3)$ & $\begin{array}{l}101 / \\
242\end{array}$ & $(41.7)$ & $\begin{array}{l}(-14.4 \\
\text { to } \\
-0.2)\end{array}$ & 0.044 & $\begin{array}{l}(-6.7 \\
\text { to } \\
9.6)\end{array}$ & 0.726 \\
\hline $\begin{array}{l}\text { Been put in sexually uncomfortable/ } \\
\text { abusive situations }\end{array}$ & $\begin{array}{l}83 / \\
566\end{array}$ & $(14.7)$ & $\begin{array}{l}163 / \\
566\end{array}$ & $(28.8)$ & $\begin{array}{l}76 / \\
324\end{array}$ & $(23.5)$ & $\begin{array}{l}144 / \\
324\end{array}$ & $(44.4)$ & $\begin{array}{l}7 / \\
242\end{array}$ & (2.9) & $\begin{array}{l}19 / \\
242\end{array}$ & (7.9) & $\begin{array}{l}(15.4 \\
\text { to } \\
25.7)\end{array}$ & $\begin{array}{l}< \\
0.001\end{array}$ & $\begin{array}{l}(29.9 \\
\text { to } \\
42.7)\end{array}$ & $\begin{array}{l}< \\
0.001\end{array}$ \\
\hline
\end{tabular}

Note: ${ }^{a}$ Same questions at both $T_{1}$ and $T_{2}$ except for question "Been seriously ill", which was only asked at $T_{2}$

${ }^{b}$ Negative life events defined as "last 3 years" were events measured at $T_{2}$ only, and negative life events defined as "ever" were measured at $T_{1}$ or $T_{2}$

${ }^{c}$ Newcombe hybrid score

${ }^{d}$ Pearson Chi squared test 
Table 4 Binary linear regression with suicidal ideation at 3-year follow up as dependent variable, and negative life events as covariates

\begin{tabular}{|c|c|c|c|c|c|c|c|c|c|c|c|c|c|}
\hline \multicolumn{14}{|l|}{ Suicidal ideation $^{\mathrm{a}}$ at $\mathrm{T}_{2}$} \\
\hline \multirow{3}{*}{ Negative life events } & \multirow[b]{3}{*}{ n } & & & & & \multicolumn{3}{|c|}{ Crude } & \multirow[b]{3}{*}{$\begin{array}{l}p \\
\text { value }\end{array}$} & \multicolumn{4}{|c|}{ Adjusted for SES } \\
\hline & & \multicolumn{2}{|c|}{$\begin{array}{l}\text { No Neg. } \\
\text { life event }\end{array}$} & \multicolumn{2}{|c|}{$\begin{array}{l}\text { Neg. life } \\
\text { event }\end{array}$} & \multirow{2}{*}{$\begin{array}{l}\mathbf{R D}^{\mathbf{b}} \\
\%\end{array}$} & \multicolumn{2}{|c|}{$95 \% \mathrm{Cl}$ for $\mathrm{RD}$} & & \multirow{2}{*}{$\begin{array}{l}\text { RD } \\
\%\end{array}$} & \multicolumn{2}{|c|}{$95 \% \mathrm{Cl}$ for $\mathrm{RD}$} & \multirow[b]{2}{*}{$\begin{array}{l}p \\
\text { value }\end{array}$} \\
\hline & & $n$ & $(\%)$ & $n$ & (\%) & & Lower & Upper & & & Lower & Upper & \\
\hline Total sample & 549 & & & & & & & & & & & & \\
\hline $\begin{array}{l}\text { Serious illness of someone in family or death of a loved } \\
\text { one }\end{array}$ & 535 & $\begin{array}{l}3 / \\
73^{c}\end{array}$ & $(4.1)$ & $\begin{array}{l}63 / \\
462^{c}\end{array}$ & $(13.6)$ & 9.5 & 4.0 & 15.1 & 0.001 & 8.1 & 0.9 & 15.2 & 0.027 \\
\hline $\begin{array}{l}\text { Been seriously ill or injured, received painful or } \\
\text { frightening treatment at hospital }\end{array}$ & 536 & $\begin{array}{l}39 / \\
367\end{array}$ & $(10.6)$ & $\begin{array}{l}27 / \\
169\end{array}$ & $(16.0)$ & 5.3 & -1.0 & 11.7 & 0.100 & 8.6 & 0.9 & 16.3 & 0.028 \\
\hline Exposed to a serious accident or catastrophe & 536 & $\begin{array}{l}40 / \\
365\end{array}$ & $(11.0)$ & $\begin{array}{l}26 / \\
171\end{array}$ & $(15.2)$ & 4.2 & -2.0 & 10.5 & 0.184 & 5.5 & -2.2 & 13.3 & 0.160 \\
\hline Been threatened, physically harassed or violently hurt & 536 & $\begin{array}{l}26 / \\
287\end{array}$ & $(9.1)$ & $\begin{array}{l}40 / \\
249\end{array}$ & $(16.1)$ & 7.0 & 1.4 & 12.7 & 0.015 & 8.9 & 2.0 & 15.9 & 0.012 \\
\hline Seen others violently hurt & 536 & $\begin{array}{l}32 / \\
306\end{array}$ & $(10.5)$ & $\begin{array}{l}34 / \\
230\end{array}$ & $(14.8)$ & 4.3 & -1.4 & 10.1 & 0.139 & 5.7 & -1.4 & 12.7 & 0.115 \\
\hline Been put in sexually uncomfortable/abusive situations & 536 & $\begin{array}{l}36 / \\
383\end{array}$ & $(9.4)$ & $\begin{array}{l}30 / \\
153\end{array}$ & $(19.6)$ & 10.2 & 3.3 & 17.2 & 0.004 & 10.4 & 1.8 & 19.0 & 0.018 \\
\hline SES & 385 & & & & & $0.2^{d}$ & -1.7 & 2.2 & 0.820 & & & & \\
\hline Girls & 308 & & & & & & & & & & & & \\
\hline $\begin{array}{l}\text { Serious illness of someone in family or death of a loved } \\
\text { one }\end{array}$ & 304 & $2 / 35$ & $(5.7)$ & $\begin{array}{l}52 / \\
269\end{array}$ & $(19.3)$ & 13.6 & 4.6 & 22.6 & 0.003 & 12.7 & 0.8 & 24.5 & 0.037 \\
\hline $\begin{array}{l}\text { Been seriously ill or injured, received painful or } \\
\text { frightening treatment at hospital }\end{array}$ & 305 & $\begin{array}{l}30 / \\
188\end{array}$ & $(16.0)$ & $\begin{array}{l}24 / \\
117\end{array}$ & $(20.5)$ & 4.6 & -4.5 & 13.6 & 0.322 & 8.4 & -2.7 & 19.6 & 0.138 \\
\hline Exposed to a serious accident or catastrophe & 305 & $\begin{array}{l}31 / \\
192\end{array}$ & $(16.2)$ & $\begin{array}{l}23 / \\
113\end{array}$ & $(20.4)$ & 4.2 & -4.9 & 13.3 & 0.364 & 7.2 & -4.3 & 18.7 & 0.221 \\
\hline Been threatened, physically harassed or violently hurt & 305 & $\begin{array}{l}21 / \\
153\end{array}$ & $(13.7)$ & $\begin{array}{l}33 / \\
152\end{array}$ & $(21.7)$ & 8.0 & -0.5 & 16.5 & 0.067 & 11.1 & 0.2 & 21.9 & 0.045 \\
\hline Seen others violently hurt & 305 & $\begin{array}{l}25 / \\
170\end{array}$ & $(14.7)$ & $\begin{array}{l}29 / \\
135\end{array}$ & $(21.5)$ & 6.8 & -2.0 & 15.5 & 0.129 & 9.1 & -2.0 & 20.2 & 0.109 \\
\hline Been put in sexually uncomfortable/abusive situations & 305 & $\begin{array}{l}27 / \\
170\end{array}$ & $(15.9)$ & $\begin{array}{l}27 / \\
135\end{array}$ & $(20.0)$ & 4.1 & -4.6 & 12.8 & 0.354 & 6.1 & -4.9 & 17.0 & 0.277 \\
\hline SES & 210 & & & & & -0.4 & -3.6 & 2.8 & 0.806 & & & & \\
\hline Boys & 241 & & & & & & & & & & & & \\
\hline $\begin{array}{l}\text { Serious illness of someone in family or death of a loved } \\
\text { one }\end{array}$ & 231 & $1 / 38$ & $(2.6)$ & $\begin{array}{l}11 / \\
193\end{array}$ & $(5.7)$ & 3.1 & -3.0 & 9.1 & 0.321 & 0.9 & -6.8 & 8.6 & 0.821 \\
\hline $\begin{array}{l}\text { Been seriously ill or injured, received painful or } \\
\text { frightening treatment at hospital }\end{array}$ & 231 & $\begin{array}{l}9 / \\
179\end{array}$ & $(5.0)$ & $3 / 52$ & $(5.8)$ & 0.7 & -6.4 & 7.9 & 0.838 & 3.9 & -4.9 & 12.7 & 0.387 \\
\hline Exposed to a serious accident or catastrophe & 231 & $\begin{array}{l}9 / \\
173\end{array}$ & $(5.2)$ & $3 / 58$ & $(5.2)$ & 0.0 & -6.6 & 6.6 & 0.993 & 3.0 & -8.8 & 2.8 & 0.308 \\
\hline Been threatened, physically harassed or violently hurt & 231 & $\begin{array}{l}5 / \\
134\end{array}$ & $(3.7)$ & $7 / 97$ & $(7.2)$ & 3.5 & -2.6 & 9.6 & 0.261 & 3.6 & -3.5 & 10.7 & 0.317 \\
\hline Seen others violently hurt & 231 & $\begin{array}{l}7 / \\
136\end{array}$ & $(5.2)$ & $5 / 95$ & $(5.3)$ & 0.1 & -5.7 & 6.0 & 0.969 & 0.8 & -5.9 & 7.4 & 0.820 \\
\hline Been put in sexually uncomfortable/abusive situations & 231 & $\begin{array}{l}9 / \\
213\end{array}$ & $(4.2)$ & $3 / 18$ & $(16.7)$ & 12.4 & -5.0 & 29.9 & 0.163 & 3.2 & -11.0 & 17.4 & 0.659 \\
\hline SES & 175 & & & & & 1.1 & -0.1 & 2.4 & 0.072 & & & & \\
\hline
\end{tabular}

Note: Binary linear regression is based on paired data displayed in Supplemental Material Table S2. SES Socioeconomic status

a Suicidal ideation includes suicidal thoughts occasionally or often

${ }^{b} R D$ is risk difference, the difference between the proportions of patients with suicidal thoughts or behavior and negative life events compared with patients with suicidal thoughts or behavior without negative life event

"The numbers in this table, for example 3/73 (4.1) and 63/462 (13.6), indicate that among the 73 patients with no negative life event, 3 had suicidal ideation at $T_{2}$, and among the 462 patients with the negative life event, 63 had suicidal ideation at T2

${ }^{d}$ The risk of having suicidal ideation increases with $0.2 \%$ per one unit increase in level of mothers education 
Table 5 Binary linear regression with suicidal behavior at 3-year follow up as dependent variable, and negative life events as covariates

\begin{tabular}{|c|c|c|c|c|c|c|c|c|c|c|c|c|c|}
\hline \multicolumn{14}{|l|}{ Suicidal behavior $^{\mathrm{a}}$ at $\mathrm{T}_{2}$} \\
\hline \multirow{3}{*}{ Negative life events } & \multirow[b]{3}{*}{$\mathrm{n}$} & & & & & \multicolumn{3}{|c|}{ Crude } & \multirow[b]{3}{*}{$\begin{array}{l}p \\
\text { value }\end{array}$} & \multicolumn{4}{|c|}{ Adjusted for SES } \\
\hline & & \multicolumn{2}{|c|}{$\begin{array}{l}\text { No Neg. } \\
\text { life event }\end{array}$} & \multicolumn{2}{|c|}{$\begin{array}{l}\text { Neg. life } \\
\text { event }\end{array}$} & \multirow{2}{*}{$\begin{array}{l}\mathrm{RD}^{\mathrm{b}} \\
\%\end{array}$} & \multicolumn{2}{|c|}{$95 \% \mathrm{Cl}$ for RD } & & \multirow{2}{*}{$\begin{array}{l}\mathrm{RD} \\
\%\end{array}$} & \multicolumn{2}{|c|}{$95 \% \mathrm{Cl}$ for $\mathrm{RD}$} & \multirow[b]{2}{*}{$\begin{array}{l}p \\
\text { value }\end{array}$} \\
\hline & & $\bar{n}$ & $(\%)$ & $\mathrm{n}$ & (\%) & & Lower & Upper & & & Lower & Upper & \\
\hline Total sample & 549 & & & & & & & & & & & & \\
\hline Serious illness of someone in family or death of a loved one & 536 & $\begin{array}{l}8 / \\
73^{c}\end{array}$ & $(11.0)$ & $\begin{array}{l}89 / \\
463^{c}\end{array}$ & $(19.2)$ & 8.3 & -0.2 & 16.3 & 0.043 & $-{ }^{d}$ & - & - & - \\
\hline $\begin{array}{l}\text { Been seriously ill or injured, received painful or frightening } \\
\text { treatment at hospital }\end{array}$ & 537 & $\begin{array}{l}50 / \\
368\end{array}$ & $(13.6)$ & $\begin{array}{l}48 / \\
169\end{array}$ & $(28.4)$ & 14.8 & 7.2 & 22.5 & $\begin{array}{l}< \\
0.001\end{array}$ & 10.6 & 2.8 & 18.4 & 0.008 \\
\hline Exposed to a serious accident or catastrophe & 537 & $\begin{array}{l}52 / \\
365\end{array}$ & $(14.2)$ & $\begin{array}{l}46 / \\
172\end{array}$ & $(26.7)$ & 12.5 & 5.0 & 20.0 & 0.001 & 10.1 & 1.9 & 18.3 & 0.015 \\
\hline Been threatened, physically harassed or violently hurt & 537 & $\begin{array}{l}28 / \\
287\end{array}$ & $(9.8)$ & $\begin{array}{l}70 / \\
250\end{array}$ & $(28.0)$ & 18.2 & 11.7 & 24.8 & $\begin{array}{l}< \\
0.001\end{array}$ & 16.7 & 9.5 & 23.9 & $\begin{array}{l}< \\
0.001\end{array}$ \\
\hline Seen others violently hurt & 537 & $\begin{array}{l}37 / \\
306\end{array}$ & $(12.1)$ & $\begin{array}{l}61 / \\
231\end{array}$ & $(26.4)$ & 14.3 & 7.6 & 21.1 & $\begin{array}{l}< \\
0.001\end{array}$ & 10.7 & 3.4 & 18.1 & 0.004 \\
\hline Been put in sexually uncomfortable/abusive situations & 537 & $\begin{array}{l}46 / \\
383\end{array}$ & $(12.0)$ & $\begin{array}{l}52 / \\
154\end{array}$ & $(33.8)$ & 21.8 & 13.6 & 29.9 & $\begin{array}{l}< \\
0.001\end{array}$ & - & - & - & - \\
\hline SES & 386 & & & & & $-2.3^{\mathrm{e}}$ & -4.4 & -0.8 & 0.005 & & & & \\
\hline Girls & 308 & & & & & & & & & & & & \\
\hline Serious illness of someone in family or death of a loved one & 305 & $7 / 35$ & $(20.0)$ & $\begin{array}{l}68 / \\
270\end{array}$ & $(25.2)$ & 5.2 & -9.1 & 19.4 & 0.476 & - & - & - & - \\
\hline $\begin{array}{l}\text { Been seriously ill or injured, received painful or frightening } \\
\text { treatment at hospital }\end{array}$ & 306 & $\begin{array}{l}37 / \\
189\end{array}$ & $(19.6)$ & $\begin{array}{l}39 / \\
117\end{array}$ & $(33.3)$ & 13.8 & 3.5 & 24.0 & 0.009 & 7.4 & -3.5 & 18.3 & 0.184 \\
\hline Exposed to a serious accident or catastrophe & 306 & $\begin{array}{l}39 / \\
192\end{array}$ & $(20.3)$ & $\begin{array}{l}37 / \\
114\end{array}$ & $(32.5)$ & 12.1 & 1.8 & 22.5 & 0.021 & 6.3 & -5.1 & 17.6 & 0.280 \\
\hline Been threatened, physically harassed or violently hurt & 306 & $\begin{array}{l}22 / \\
153\end{array}$ & $(14.4)$ & $\begin{array}{l}54 / \\
153\end{array}$ & $(35.3)$ & 20.9 & 11.5 & 30.3 & $\begin{array}{l}< \\
0.001\end{array}$ & 17.6 & 6.9 & 28.3 & 0.001 \\
\hline Seen others violently hurt & 306 & $\begin{array}{l}27 / \\
170\end{array}$ & $(15.9)$ & $\begin{array}{l}49 / \\
136\end{array}$ & $(36.0)$ & 20.1 & 10.4 & 29.9 & $\begin{array}{l}< \\
0.001\end{array}$ & 14.6 & 3.4 & 25.8 & 0.011 \\
\hline Been put in sexually uncomfortable/abusive situations & 306 & $\begin{array}{l}27 / \\
170\end{array}$ & $(15.9)$ & $\begin{array}{l}49 / \\
136\end{array}$ & $(36.0)$ & 20.1 & 10.4 & 29.9 & $\begin{array}{l}< \\
0.001\end{array}$ & - & - & - & - \\
\hline SES & 211 & & & & & -3.6 & -6.5 & 0.6 & 0.017 & & & & \\
\hline Boys & 241 & & & & & & & & & & & & \\
\hline Serious illness of someone in family or death of a loved one & 231 & $1 / 38$ & $(2.6)$ & $\begin{array}{l}21 / \\
193\end{array}$ & $(10.9)$ & 8.3 & 1.5 & 15.0 & 0.016 & - & - & - & - \\
\hline $\begin{array}{l}\text { Been seriously ill or injured, received painful or frightening } \\
\text { treatment at hospital }\end{array}$ & 231 & $\begin{array}{l}13 / \\
179\end{array}$ & $(7.3)$ & $9 / 52$ & $(17.3)$ & 10.0 & -0.9 & 21.0 & 0.073 & - & - & - & - \\
\hline Exposed to a serious accident or catastrophe & 231 & $\begin{array}{l}13 / \\
173\end{array}$ & $(7.5)$ & $9 / 58$ & $(15.5)$ & 8.0 & -2.1 & 18.1 & 0.122 & 11.1 & -0.4 & 22.7 & 0.152 \\
\hline Been threatened, physically harassed or violently hurt & 231 & $\begin{array}{l}6 / \\
134\end{array}$ & $(4.5)$ & $16 / 97$ & $(16.5)$ & 12.0 & 3.8 & 20.2 & 0.004 & - & - & - & - \\
\hline Seen others violently hurt & 231 & $\begin{array}{l}10 / \\
136\end{array}$ & $(7.4)$ & $12 / 95$ & $(12.6)$ & 5.3 & -2.7 & 13.3 & 0.196 & 5.1 & -2.8 & 13.0 & 0.206 \\
\hline Been put in sexually uncomfortable/abusive situations & 231 & $\begin{array}{l}19 / \\
213\end{array}$ & (8.9) & $3 / 18$ & (16.7) & 7.7 & -9.9 & 25.4 & 0.390 & 15.7 & -5.8 & 37.2 & 0.152 \\
\hline SES & 175 & & & & & -1.7 & -3.4 & -0.0 & 0.047 & & & & \\
\hline
\end{tabular}

Note: Binary linear regression is based on paired data displayed in Supplemental Material Table S3. SES Socioeconomic status

a Suicidal behavior includes suicidal acts or attempts, presently at $T_{2}$ or ever, also suicidal acts and attempts with suicidal thoughts

${ }^{b} R D$ is risk difference, the difference between the proportions of patients with suicidal thoughts or behavior and negative life events compared with patients with suicidal thoughts or behavior without negative life event

${ }^{c}$ The numbers in this table, for example 3/73 (4.1) and 63/462 (13.6), indicate that among the 73 patients with no negative life event, 3 had suicidal ideation at $T_{2}$, and among the 462 patients with the negative life event, 63 had suicidal ideation at $T 2$

${ }^{d}$ Estimates could not be computed due to non-convergence of the calculations

e The risk of having suicidal behavior decreases with $2.3 \%$ per one unit increase in level of mothers education 
disorders and Anxiety disorders $(p<0.001)$ and the group Other disorders $(p=0.007)$, adjusted for SES (data not shown).

There were associations between school dropout and having seen others been violently hurt or been put in sexually uncomfortable/abusive situations, but after adjusting for SES, the associations only persisted for having seen others been violently hurt $(\mathrm{RD}=10.8 \%$, CI $(2.9$ to 18.8$), p=0.007)$, and only among girls $(\mathrm{RD}=11.7 \%$, CI (0.5 to 22.9), $p=0.041$ ) (Table 6). Results were mainly unchanged when excluding those who gave childbirth $(n=6)$ (data not shown). An association found between suicidal behavior and school dropout was attenuated after adjustment for SES in the total sample (RD $=7.0 \%$, CI ( -3.9 to 17.9$), p=0.209$ ).

\section{Discussion}

This study is one of few surveys following a general clinical psychiatric population of adolescents who received standard clinical care, studying symptoms and function over time. Reassessed after 3 years, suicidal ideation and suicidal behavior were frequent, especially among girls, and across all subgroups of psychiatric disorders. Girls had lower psychosocial functioning than boys, and more school dropout. Associations were found between negative life events and suicidal ideation and behavior. Most marked were the associations between suicidal behavior and having been exposed to interpersonal violence for both girls and boys. For girls only, sexually uncomfortable or abusive situations were also related to suicidal ideation and suicidal behavior, as was having seen others been violently hurt. Furthermore, having serious illness of someone in family or death of a loved one were also associated with suicidal ideation for the total sample, and SES was linked to suicidal behavior for both girls and boys. School dropout was associated with having seen others be violently hurt among girls with ADHD.

In our sample assessed 3 years after referral for psychiatric disorders, the prevalence of suicidal ideation and behavior were similar to earlier research on clinical samples $[7,17]$. The frequent occurrence of suicidal attempts may reflect that this is a follow up of former patients, with high rates of psychiatric disorders [46], and the results correspond well with earlier research describing that the majority of youth with suicidal behaviors have pre-existing mental disorders [5]. Still, the reasons for the high rates of suicidal ideation and behavior may be diverse, both depending on the persistence of psychiatric disorders [46], treatment given and the general vulnerability of the adolescents in this clinical population. There were large gender differences with girls having much higher rates than boys of both suicidal ideation and behavior, in line with earlier research [5], and especially described in the systematic review of 67 population-based longitudinal studies with focus on gender differences in suicidal behavior in adolescents and young adults [14]. In our study, almost one out of two girls with mood disorders had both suicidal ideation and suicidal behavior, whereas less than one out of four boys with mood disorders had the same symptoms. Boys with ADHD or other psychiatric disorders had the lowest frequencies of suicidal ideation or behavior. This follow up of former adolescent patients underscores the large gender differences and added risk for girls when it comes to suicidal symptoms.

Psychosocial function as measured by CGAS with values in the sub-normal range, indicated better functioning than expected in a clinical sample with frequent comorbidity. The inter-rater reliability was tested and shown to be good. CGAS was lower among girls than among boys, corresponding with earlier findings by Gårdvik et al. [46], showing that female participants had higher rates of psychiatric disorders and seemed to be more prone to develop co-occurring psychiatric disorders and a higher burden of disease. School dropout was also significantly higher among girls compared to boys, which may once again reflect a higher burden of disease among girls in this sample. Earlier research has showed that poor health, and especially mental health, has been significantly associated with dropout of school among adolescents, most marked for boys in higher education $[33,36]$. Development and persistence of psychiatric disorders is prone to impact function in school and socialization, with possible long-term consequences [2]. It is therefore crucial to break the cycle at an early stage and hinder maintenance of problems.

The frequencies of experiencing negative life events are in accordance with earlier research reporting associations between psychiatric disorders and earlier negative life events or childhood adversities [54-56]. The most common experiences, in both genders, were serious illness of someone in the family or death of a loved one, reported for eight to nine out of ten adolescents. Almost half of the adolescents had been threatened, physically harassed or violently hurt, or seen others violently hurt, with no gender differences, whereas there were large gender differences in exposure to sexually uncomfortable or abusive situations. Less than one out of ten boys had such experiences, but almost one out of two girls were exposed. The results underline the importance of assessing negative life events among adolescents with psychiatric symptoms and disorders, in order to reveal any such risks, give proper treatment and if possible, prevent further traumatic events.

We examined possible associations between suicidal ideation at follow-up and negative life events. There was a significant association between suicidal ideation and having been threatened, physically harassed or violently 
Table 6 Binary linear regression with school dropout at 3-year follow up as dependent variable, and negative life events as covariate

\begin{tabular}{|c|c|c|c|c|c|c|c|c|c|c|c|c|c|}
\hline \multicolumn{14}{|l|}{ School dropout ${ }^{\mathrm{a}}$ at $\mathrm{T}_{2}$} \\
\hline \multirow{3}{*}{ Negative life events } & \multirow[b]{3}{*}{$\mathbf{n}$} & & & & & \multicolumn{3}{|c|}{ Crude } & \multirow[b]{3}{*}{$\begin{array}{l}p \\
\text { value }\end{array}$} & \multicolumn{4}{|c|}{ Adjusted for SES } \\
\hline & & \multicolumn{2}{|c|}{$\begin{array}{l}\text { No Neg. } \\
\text { life event }\end{array}$} & \multicolumn{2}{|c|}{$\begin{array}{l}\text { Neg. life } \\
\text { event }\end{array}$} & \multirow{2}{*}{$\begin{array}{l}\mathbf{R D}^{\mathbf{b}} \\
\%\end{array}$} & \multicolumn{2}{|c|}{$95 \% \mathrm{Cl}$ for $\mathrm{RD}$} & & \multirow{2}{*}{$\begin{array}{l}\mathrm{RD} \\
\%\end{array}$} & \multicolumn{2}{|c|}{$95 \% \mathrm{Cl}$ for $\mathrm{RD}$} & \multirow[b]{2}{*}{$\begin{array}{l}p \\
\text { value }\end{array}$} \\
\hline & & $\mathrm{n}$ & (\%) & $\mathrm{n}$ & (\%) & & Lower & Upper & & & Lower & Upper & \\
\hline Total sample & 570 & & & & & & & & & & & & \\
\hline Serious illness of someone in family or death of a loved one & 541 & $\begin{array}{l}9 / \\
74^{c}\end{array}$ & $(12.2)$ & $\begin{array}{l}91 / \\
467^{c}\end{array}$ & $(19.5)$ & 7.3 & -1.0 & 15.6 & 0.083 & 5.8 & -3.3 & 15.0 & 0.213 \\
\hline $\begin{array}{l}\text { Been seriously ill or injured, received painful or frightening } \\
\text { treatment at hospital }\end{array}$ & 542 & $\begin{array}{l}67 / \\
376\end{array}$ & $(17.8)$ & $\begin{array}{l}34 / \\
166\end{array}$ & (20.5) & 2.7 & -4.6 & 9.9 & 0.472 & 3.3 & -4.8 & 11.4 & 0.424 \\
\hline Exposed to a serious accident or catastrophe & 542 & $\begin{array}{l}65 / \\
371\end{array}$ & $(17.5)$ & $\begin{array}{l}36 / \\
171\end{array}$ & $(21.0)$ & 3.5 & -3.7 & 10.8 & 0.339 & 0.9 & -7.1 & 8.9 & 0.830 \\
\hline Been threatened, physically harassed or violently hurt & 542 & $\begin{array}{l}43 / \\
289\end{array}$ & $(14.9)$ & $\begin{array}{l}58 / \\
253\end{array}$ & (22.9) & 8.0 & 1.4 & 14.7 & 0.017 & 6.2 & -1.3 & 13.8 & 0.106 \\
\hline Seen others violently hurt & 542 & $\begin{array}{l}40 / \\
312\end{array}$ & $(12.8)$ & $\begin{array}{l}61 / \\
230\end{array}$ & (26.5) & 13.7 & 6.9 & 20.5 & $\begin{array}{l}< \\
0.001\end{array}$ & 10.8 & 2.9 & 18.8 & 0.007 \\
\hline Been put in sexually uncomfortable/abusive situations & 542 & $\begin{array}{l}59 / \\
386\end{array}$ & $(15.3)$ & $\begin{array}{l}42 / \\
156\end{array}$ & $(26.9)$ & 11.6 & 3.8 & 19.5 & 0.004 & 7.4 & -1.4 & 16.3 & 0.100 \\
\hline SES & 404 & & & & & $-1.1^{d}$ & -3.2 & 1.1 & 0.319 & & & & \\
\hline Girls & 324 & & & & & & & & & & & & \\
\hline Serious illness of someone in family or death of a loved one & 310 & $5 / 35$ & $(14.3)$ & $\begin{array}{l}64 / \\
275\end{array}$ & (23.3) & 9.0 & -3.7 & 21.6 & 0.164 & 8.2 & -5.7 & 22.2 & 0.247 \\
\hline $\begin{array}{l}\text { Been seriously ill or injured, received painful or frightening } \\
\text { treatment at hospital }\end{array}$ & 311 & $\begin{array}{l}42 / \\
195\end{array}$ & $(21.5)$ & $\begin{array}{l}28 / \\
116\end{array}$ & $(24.1)$ & 2.6 & -7.1 & 12.3 & 0.600 & 3.1 & -8.1 & 14.3 & 0.588 \\
\hline Exposed to a serious accident or catastrophe & 311 & $\begin{array}{l}44 / \\
195\end{array}$ & (22.6) & $\begin{array}{l}26 / \\
116\end{array}$ & $(22.4)$ & -0.2 & -9.8 & 9.5 & 0.976 & -1.7 & -12.6 & 9.1 & 0.756 \\
\hline Been threatened, physically harassed or violently hurt & 311 & $\begin{array}{l}30 / \\
156\end{array}$ & $(19.2)$ & $\begin{array}{l}40 / \\
155\end{array}$ & (25.8) & 6.6 & -2.7 & 15.8 & 0.165 & 2.2 & -8.4 & 12.7 & 0.688 \\
\hline Seen others violently hurt & 311 & $\begin{array}{l}28 / \\
177\end{array}$ & $(15.8)$ & $\begin{array}{l}42 / \\
134\end{array}$ & (31.3) & 15.5 & 6.0 & 25.1 & 0.001 & 11.7 & 0.5 & 22.9 & 0.041 \\
\hline Been put in sexually uncomfortable/abusive situations & 311 & $\begin{array}{l}34 / \\
173\end{array}$ & $(19.7)$ & $\begin{array}{l}36 / \\
138\end{array}$ & $(26.1)$ & 6.4 & -3.0 & 15.9 & 0.181 & 3.7 & -7.2 & 14.6 & 0.505 \\
\hline SES & 221 & & & & & -1.5 & -4.8 & 1.7 & 0.351 & & & & \\
\hline Boys & 246 & & & & & & & & & & & & \\
\hline Serious illness of someone in family or death of a loved one & 231 & $4 / 39$ & $(10.3)$ & $\begin{array}{l}27 / \\
192\end{array}$ & $(14.1)$ & 3.8 & -6.9 & 14.5 & 0.487 & 2.5 & -9.2 & 14.2 & 0.674 \\
\hline $\begin{array}{l}\text { Been seriously ill or injured, received painful or frightening } \\
\text { treatment at hospital }\end{array}$ & 231 & $\begin{array}{l}25 / \\
181\end{array}$ & $(13.8)$ & $6 / 50$ & $(12.0)$ & -1.8 & -12.1 & 8.5 & 0.731 & 0.6 & -10.8 & 11.9 & 0.923 \\
\hline Exposed to a serious accident or catastrophe & 231 & $\begin{array}{l}21 / \\
176\end{array}$ & $(11.9)$ & $10 / 55$ & $(18.2)$ & 6.3 & -5.0 & 17.5 & 0.278 & 1.8 & -10.1 & 13.6 & 0.770 \\
\hline Been threatened, physically harassed or violently hurt & 231 & $\begin{array}{l}13 / \\
133\end{array}$ & $(9.8)$ & $18 / 98$ & $(18.4)$ & 8.6 & -0.6 & 17.8 & 0.067 & 9.4 & -1.5 & 20.4 & 0.092 \\
\hline Seen others violently hurt & 231 & $\begin{array}{l}12 / \\
135\end{array}$ & $(8.9)$ & $19 / 96$ & $(19.8)$ & 10.9 & 1.6 & 20.2 & 0.022 & 9.3 & -1.5 & 20.1 & 0.091 \\
\hline Been put in sexually uncomfortable/abusive situations & 231 & $\begin{array}{l}25 / \\
213\end{array}$ & $(11.7)$ & $6 / 18$ & (33.3) & 21.6 & -0.7 & 43.8 & 0.057 & 10.2 & -11.7 & 32.0 & 0.362 \\
\hline SES & 183 & & & & & -0.7 & -3.3 & 2.0 & 0.621 & & & & \\
\hline
\end{tabular}


hurt in the total sample, but in gender-specific analyses, the association was present only for girls. Suicidal ideation was also associated with exposure to sexually uncomfortable or abusive situations for the total sample, corresponding with earlier research described in the meta-analysis of 50 years of research by Franklin et al. [21]. Furthermore, we found that suicidal ideation was associated with having serious illness of someone in the family or death of a loved one, but only for girls. Losing a loved one by death may be a very stressful event for children and adolescents, and a systematic review and meta-analysis by Howarth et al., found that stressful life events increased the risk of both reported suicidal ideation and behavior [22].

Earlier studies have demonstrated associations between suicidal behavior in adolescents and experiences of negative life events, as described in the systematic review by Serafini et al. [20]. In our study, we found associations between suicidal behavior and all negative life events. There was a strong association between suicidal behavior and having been threatened, physically harassed or violently hurt, for the total sample and for both girls and boys, which is in line with previous research, as described in the meta-analysis by Castellví et al. [25]. Among adolescents, victimization by peers is highly prevalent and associated with increased risk of suicidal attempts, and the longer history of victimization, the greater risk [28]. For girls only, suicidal behavior was related to having seen others violently hurt, as reported in earlier research [27]. Suicidal behavior was furthermore associated with exposure to sexually uncomfortable or abusive situations for girls only. Sexual abuse or violence has been found to be strongly associated to suicide attempts and behavior [27], and contributing the most to suicide attempts in youths and young adults together with bullying [25]. Opposite to suicidal ideations, serious illness of someone in the family or death of a loved one, was associated with suicidal behavior for boys only. This association has been found in earlier research [26, 27], but not specified by gender. Having been seriously ill, injured or received painful or frightening treatment in hospital, was associated with suicidal behavior in the total sample, in line with the meta-analysis of 50 years of research [21]. In the WHO World Mental Health Surveys, a cross-national analysis of the associations between traumatic events and suicidal behavior were investigated, and accidents and disasters were associated with suicidal behavior [27], as also found in our study. A systematic review of population-based studies by Evans et al. [57], found that suicidal phenomena in adolescents were associated with female gender, mental health problems, negative life events and poor family functioning, corresponding well with our findings.
In our clinical sample, an association was found between suicidal behavior and SES, for both girls and boys. The risk of having suicidal behavior decreased for the total sample with $2.3 \%$ per unit increase in level of mothers' education. This indicates that SES does have an effect on the presence of suicidal behavior at followup, and that higher maternal education may be a protective factor for development of these symptoms. A large national register-based study showed strong associations between SES and suicidal risk [58].

School dropout is related to many different risk factors. A meta-analytic review by Gubbels et al. [41] described 23 risk domains with significant overall effect on school dropout, where mental health problems of the child and adverse childhood experiences were two of these domains. In our sample, we found associations between school dropout and having seen others been violently hurt, among girls only. A recent systematic review and meta-analysis of longitudinal studies by Castellví et al. [40] showed that adolescents and young adults who had school failure were at higher risk of a suicide attempt. In our clinical sample, the association found between suicidal behavior and school dropout in the crude analysis, did not withstand adjustment for SES. Therefore, SES may be a confounding factor for associations between suicidal behavior and school dropout, but may as well reflect larger $p$-values due to lower number of participants with SES information, or that the attrition was not random, i.e. that those with SES information were not representative for the entire sample.

One strength of the present study is the inclusion of a large clinical sample receiving standard clinical care, assessed after 3 years with a high response rate from $\mathrm{T}_{1}$ to $\mathrm{T}_{2}$. Furthermore, suicidal ideation and behavior were assessed in-depth by clinicians during the diagnostic interview, and not based on self-report measures which involves the limitations of less accuracy in establishing psychopathology. Some limitations need to be considered. The attrition rate was high in the initial recruitment, and even though the $T_{1}$ sample did not differ in age, gender or reason for referral compared to nonparticipants, we cannot exclude that this high attrition rate may have affected the results. Since there were more girls than boys among participants compared to nonparticipants in this study, we may have lost some of the boys with psychiatric disorders, suicidality symptoms and impaired function. Life events and school dropout were measured by self-report only. School dropout was reported by one question, and additional information would have strengthened the measure, either by using several questions or information from other sources, supplemented by asking about the subjective reasons for the respective school dropout. Family characteristics including unemployment and low socioeconomic status 
influence mental health in off-spring [59], and may have important influence on many of the negative life events measured in this study, and their associations with suicidal measures or school dropout. Using level of maternal education to indicate socioeconomic status may not enclose the complete concept of socioeconomics, and furthermore, this information was accessible for a reduced sample, which may not reflect the total study population. Also, the reduced sample resulted in reduced power in the association analyses. The fact that some estimates including suicidal behavior could not be computed due to nonconvergence of the calculations, was a statistical limitation. Treatment plausibly impact the course of suicidal ideations or behavior, also both general psychosocial function and school dropout, and information on treatment measures would have strengthened this study.

\section{Clinical implications}

The results of this study bring an important message to clinical practice. Even though clinicians know about increasing symptoms of suicidal ideation and behavior during adolescence, the self-reported high rates of suicidal attempts in this patient group should be an extra reminder, also the high rates of school dropout, especially among girls. The burden of exposure to negative life events must also be acknowledged. Comprehensive assessment of mental health problems should of course include important risk factors, and asking adolescent patients about suicidal ideation and behavior, experiences of negative life events and school functioning seems to be important, especially for female psychiatric patients, in order to reveal any such risks and prevent further traumatic events.

\section{Conclusion}

In this clinical sample reassessed after 3 years, one out of four adolescent girls with a persisting psychiatric disorder had suicidal ideations, and one out of three had a previous history of suicidal behavior. Girls had lower psychosocial functioning and higher rates of school dropout and experiences of negative life events than boys. Negative life events, especially exposure to interpersonal violence, were associated with suicidal ideation, suicidal behavior or school dropout. The high frequency of suicidal symptoms, school dropout and experiences of negative life events, indicates a high burden of challenges in functioning. The results reinforce the need to include these symptoms and associated factors in an extensive follow-up of psychiatric disorders in this age group.

\section{Supplementary Information}

The online version contains supplementary material available at https://doi. org/10.1186/s12888-021-03100-w.

Additional file 1: Table S1 Design matrix for the re-scoring of telephone interviews. Table S2 Suicidal ideation $T_{2}$ and Negative life events. Table S3 Suicidal behavior $T_{2}$ and Negative life events. Table S4 School dropout $\mathrm{T}_{2}$ and Negative life events

\section{Abbreviations}

K-SADS: Kiddie SADS: Schedule for Affective Disorders and Schizophrenia for School-Age Children; DSM-IV-TR: Diagnostic and Statistical Manual of Mental Disorders IV Text revision; ADHD: Attention Deficit Hyperactivity Disorder; CGAS: Children's Global Assessment Scale; SES: Socioeconomic status; RD: Risk difference; Cl: Confidence interval

\section{Acknowledgements}

The authors wish to acknowledge research assistant Anne Regine Sveen for coordinating data collection $\left(T_{2}\right)$, and a special gratitude is extended to the adolescents participating in the Health Survey in Department of Children and Youth, Division of Mental Health Care, St. Olavs hospital, Trondheim University Hospital, Norway.

\section{Authors' contributions}

KSG reviewed the literature, drafted and revised the manuscript, performed and interpreted statistical analyses, with substantial contribution by MSI in the entire process. SL contributed to performing and interpreting the statistical analyses, in addition to being involved in revising the manuscript. $\Pi T$ and MR participated in designing the study and made substantial contributions in revising the manuscript critically. All authors read and approved the final manuscript.

\section{Funding}

The Liaison Committee for education, research and innovation in Central Norway awarded a PhD grant to the first author. The Health Survey in Department of Children and Youth, Division of Mental Health Care, St. Olavs hospital, Trondheim University Hospital, Norway (St. Olav CAP Survey), is run in professional and financial collaboration between St. Olavs hospital and the Regional Centre for Child and Youth Mental Health and Child Welfare, Department of Mental Health, Faculty of Medicine and Health Sciences, Norwegian University of Science and Technology (NTNU). Additional funding was assigned from The Liaison Committee for education, research and innovation, Central Norway $\left(\mathrm{T}_{1}\right)$ and from the Joint Research Committee between St. Olavs hospital and Faculty of Medicine and Health Sciences, $\operatorname{NTNU}\left(\mathrm{T}_{2}\right)$

\section{Availability of data and materials}

The datasets analyzed during the current study are not publicly available due to privacy policy, but they are available from the corresponding author on reasonable request.

\section{Ethics approval and consent to participate}

Written informed consent was obtained from adolescents and parents participating at $T_{1}$ and $T_{2}$, according to study procedures. Study approval was given by the Regional Committee for Medical and Health Research Ethics of Central Norway (REK Midt) (reference numbers CAP survey $T_{1}$ : 4.2008.1393, $T_{2}:$ 2011/1435/REK Midt, and present study using $T_{1}$ and $T_{2}$ data: 2017/589/REK Midt). The Norwegian Social Science Data Services, The Data Protection Official for Research, gave permission to compare the main reason for referral, age and sex between participants and non-participants at baseline (reference number CAP survey: 19976). The research followed the agreement of the ethical standards in the Declaration of Helsinki from 1964 and later amendments, and we confirm that all methods were performed in accordance with relevant guidelines and regulations.

\section{Consent for publication}

Not applicable.

\section{Competing interests}

The authors declare that they have no competing interest. 


\section{Author details}

${ }^{1}$ Regional Centre for Child and Youth Mental Health and Child Welfare, Department of Mental Health, Faculty of Medicine and Health Sciences, Norwegian University of Science and Technology, Trondheim, Norway. ${ }^{2}$ Department of Children and Youth, Division of Mental Health Care, St. Olavs Hospital, Trondheim University Hospital, Trondheim, Norway. ${ }^{3}$ Orkdal District Psychiatric Centre, Division of Mental Health Care, St. Olavs hospital, Trondheim University Hospital, Trondheim, Norway. ${ }^{4}$ Department of Mental Health, Faculty of Medicine and Health Sciences, Norwegian University of Science and Technology, Trondheim, Norway. ${ }^{5}$ Department of Clinical and Molecular Medicine, Faculty of Medicine and Health Sciences, Norwegian University of Science and Technology, Trondheim, Norway. ${ }^{6}$ Department of Pediatrics, St. Olavs hospital, Trondheim University Hospital, Trondheim, Norway.

Received: 4 January 2021 Accepted: 25 January 2021

Published online: 18 February 2021

\section{References}

1. Patton GC, Coffey C, Sawyer SM, Viner RM, Haller DM, Bose K, et al. Global patterns of mortality in young people: a systematic analysis of population health data. Lancet. 2009;374(9693):881-92.

2. de Girolamo G, Dagani J, Purcell R, Cocchi A, McGorry PD. Age of onset of mental disorders and use of mental health services: needs, opportunities and obstacles. Epidemiol Psychiatr Sci. 2012;21(1):47-57.

3. Gore FM, Bloem PJ, Patton GC, Ferguson J, Joseph V, Coffey C, et al. Global burden of disease in young people aged 10-24 years: a systematic analysis. Lancet. 2011;377(9783):2093-102. https://doi.org/10.1016/s0140-6736(11 60512-6.

4. Nock MK, Borges G, Bromet EJ, Cha CB, Kessler RC, Lee S. Suicide and suicidal behavior. Epidemiol Rev. 2008:30(1):133-54.

5. Nock MK, Green JG, Hwang I, McLaughlin KA, Sampson NA, Zaslavsky AM, et al. Prevalence, correlates, and treatment of lifetime suicidal behavior among adolescents: results from the National Comorbidity Survey Replication Adolescent Supplement. JAMA Psychiat. 2013;70(3):300-10.

6. Turecki G, Brent DA. Suicide and suicidal behaviour. Lancet. 2016;387(10024): 1227-39.

7. Glenn CR, Lanzillo EC, Esposito EC, Santee AC, Nock MK, Auerbach RP. Examining the course of suicidal and nonsuicidal self-injurious thoughts and behaviors in outpatient and inpatient adolescents. J Abnorm Child Psychol. 2017:45(5):971-83.

8. Mars B, Heron J, Klonsky ED, Moran P, O'Connor RC, Tilling K, et al. Predictors of future suicide attempt among adolescents with suicidal thoughts or non-suicidal self-harm: a population-based birth cohort study. Lancet Psychiatry. 2019;6(4):327-37.

9. Brezo J, Paris J, Barker ED, Tremblay R, Vitaro F, Zoccolillo M, et al. Natural history of suicidal behaviors in a population-based sample of young adults. Psychol Med. 2007;37(11):1563-74.

10. Tørmoen AJ, Myhre M, Walby FA, Grøholt B, Rossow I. Changes in prevalence of self-harm from 2002 to 2018 among Norwegian adolescents. Eur J Public Health. 2020;30(1):688-92.

11. Hawton $\mathrm{K}, \mathrm{O}^{\prime}$ Connor RC. Self-harm in adolescence and future mental health Lancet. 2012;379(9812):198-9. https://doi.org/10.1016/s0140-6736(11)612609.

12. Glenn CR, Kleiman EM, Kellerman J, Pollak O, Cha CB, Esposito EC, et al. Annual research review: a meta-analytic review of worldwide suicide rates in adolescents. J Child Psychol Psychiatry. 2020;61(3):294-308.

13. World Health Organization. Suicide in the world: global health estimates. World Health Organization; 2019. https://apps.who.int/iris/handle/10665/32 6948.

14. Miranda-Mendizabal A, Castellví P, Parés-Badell O, Alayo I, Almenara J, Alonso I, et al. Gender differences in suicidal behavior in adolescents and young adults: systematic review and meta-analysis of longitudinal studies. Int J Public Health. 2019;64(2):265-83.

15. Miranda-Mendizabal A, Castellví P, Alayo I, Vilagut G, Blasco MJ, Torrent A, et al. Gender commonalities and differences in risk and protective factors of suicidal thoughts and behaviors: a cross-sectional study of Spanish university students. Depress Anxiety. 2019;36(11):1102-14.

16. Costello EJ, Copeland W, Angold A. Trends in psychopathology across the adolescent years: what changes when children become adolescents, and when adolescents become adults? J Child Psychol Psychiatry. 2011;52(10): 1015-25. https://doi.org/10.1111/j.1469-7610.2011.02446.x.

17. Asarnow JR, Porta G, Spirito A, Emslie G, Clarke G, Wagner KD, et al. Suicide attempts and nonsuicidal self-injury in the treatment of resistant depression in adolescents: findings from the TORDIA study. J Am Acad Child Adolesc Psychiatry. 2011;50(8):772-81.

18. Felitti VJ, Anda RF. The relationship of adverse childhood experiences to adult medical disease, psychiatric disorders, and sexual behavior: implications for healthcare. Impact Early Life Trauma Health Dis. 2010:77-87. https://doi.org/10.1017/CBO9780511777042.010.

19. Copeland WE, Keeler G, Angold A, Costello EJ. Traumatic events and posttraumatic stress in childhood. Arch Gen Psychiatry. 2007;64(5):577-84

20. Serafini G, Muzio C, Piccinini G, Flouri E, Ferrigno G, Pompili M, et al. Life adversities and suicidal behavior in young individuals: a systematic review. Eur Child Adolesc Psychiatry. 2015;24(12):1423-46.

21. Franklin JC, Ribeiro JD, Fox KR, Bentley KH, Kleiman EM, Huang X, et al. Risk factors for suicidal thoughts and behaviors: a meta-analysis of 50 years of research. Psychol Bull. 2017;143(2):187.

22. Howarth EJ, O'Connor DB, Panagioti M, Hodkinson A, Wilding S, Johnson J. Are stressful life events prospectively associated with increased suicidal ideation and behaviour? A systematic review and meta-analysis. J Affect Disord. 2020;266:731-42. https://doi.org/10.1016/j.jad.2020.01.171.

23. Stoltenborgh M, Van ljzendoorn MH, Euser EM, Bakermans-Kranenburg MJ. A global perspective on child sexual abuse: meta-analysis of prevalence around the world. Child Maltreat. 2011;16(2):79-101.

24. Reigstad B, Jørgensen K, Wichstrøm L. Diagnosed and self-reported childhood abuse in national and regional samples of child and adolescent psychiatric patients: Prevalences and correlates. Nord J Psychiatry. 2006;60(1):58-66.

25. Castellví P, Miranda-Mendizábal A, Parés-Badell O, Almenara J, Alonso I, Blasco M, et al. Exposure to violence, a risk for suicide in youths and young adults. A metaanalysis of longitudinal studies. Acta Psychiatr Scand. 2017;135(3):195-211.

26. Jakobsen IS, Christiansen E. Young people's risk of suicide attempts in relation to parental death: a population-based register study. J Child Psychol Psychiatry. 2011:52(2):176-83.

27. Stein DJ, Chiu WT, Hwang I, Kessler RC, Sampson N, Alonso J, et al. Crossnational analysis of the associations between traumatic events and suicidal behavior: findings from the WHO world mental health surveys. PLOS One. 2010:5(5):e10574.

28. Geoffroy M-C, Boivin M, Arseneault L, Turecki G, Vitaro F, Brendgen M, et al. Associations between peer victimization and suicidal ideation and suicide attempt during adolescence: results from a prospective population-based birth cohort. J Am Acad Child Adolesc Psychiatry. 2016;55(2):99-105.

29. Liu X, Chen H, Liu Z, Wang J, Jia C. Prevalence of suicidal behaviour and associated factors in a large sample of Chinese adolescents. Epidemiol Psychiatr Sci. 2019;28(3):280-9.

30. Zhang J, Ma Z. Patterns of life events preceding the suicide in rural young Chinese: a case control study. J Affect Disord. 2012;140(2):161-7.

31. Danese A, McEwen BS. Adverse childhood experiences, allostasis, allostatic load, and age-related disease. Physiol Behav. 2012;106(1):29-39.

32. Greger HK, Myhre AK, Lydersen S, Jozefiak T. Previous maltreatment and present mental health in a high-risk adolescent population. Child Abuse Negl. 2015;45:122-34.

33. De Ridder KA, Pape K, Johnsen R, Westin S, Holmen TL, Bjorngaard JH. School dropout: a major public health challenge: a 10-year prospective study on medical and non-medical social insurance benefits in young adulthood, the young-HUNT 1 study (Norway). J Epidemiol Community Health. 2012;66(11):995-1000. https://doi.org/10.1136/jech-2011-200047.

34. Sagatun $\AA$, Heyerdahl S, Wentzel-Larsen T, Lien L. Mental health problems in the 10 th grade and non-completion of upper secondary school: the mediating role of grades in a population-based longitudinal study. BMC Public Health. 2014;14(1):16.

35. World Health Organization. Education and health go hand in hand; 2010. Retrieved April1, 2020 from https://www.whoint/dg/speeches/2010/educa tionandhealth 20100920/en/

36. Hjorth CF, Bilgrav L, Frandsen LS, Overgaard C, Torp-Pedersen C, Nielsen B, et al. Mental health and school dropout across educational levels and genders: a 4.8-year follow-up study. BMC Public Health. 2016;16:976. https:// doi.org/10.1186/s12889-016-3622-8.

37. Dupéré V, Dion E, Leventhal T, Archambault I, Crosnoe R, Janosz M. High school dropout in proximal context: the triggering role of stressful life events. Child Dev. 2018;89(2):e107-22. 
38. Eckhoff C, Sørvold MT, Kvernmo S. Adolescent self-harm and suicidal behavior and young adult outcomes in indigenous and non-indigenous people. Eur Child Adolesc Psychiatry. 2020;29:917-27.

39. Copeland WE, Goldston DB, Costello EJ. Adult associations of childhood suicidal thoughts and behaviors: a prospective, longitudinal analysis. J Am Acad Child Adolesc Psychiatry. 2017:56(11):958-965. e954.

40. Castellví P, Miranda-Mendizábal A, Alayo I, Parés-Badell O, Almenara J, Alonso I, et al. Assessing the relationship between school failure and suicidal behavior in adolescents and young adults: a systematic review and meta-analysis of longitudinal studies. Sch Ment Heal. 2020;12:429-41.

41. Gubbels J, van der Put CE, Assink M. Risk factors for school absenteeism and dropout: a meta-analytic review. J Youth Adolesc. 2019;48(9):1637-67.

42. McDermott ER, Donlan AE, Zaff JF. Why do students drop out? Turning points and long-term experiences. J Educ Res. 2019;112(2):270-82.

43. Samuel R, Burger K. Negative life events, self-efficacy, and social support: risk and protective factors for school dropout interventions and dropout. J Educ Psychol. 2020;112(5):973-86.

44. Lavoie L, Dupéré V, Dion E, Crosnoe R, Lacourse E, Archambault I. Gender differences in adolescents' exposure to stressful life events and differential links to impaired school functioning. J Abnorm Child Psychol. 2019;47(6): 1053-64.

45. Mangerud WL, Bjerkeset O, Lydersen S, Indredavik MS. Chronic pain and pain-related disability across psychiatric disorders in a clinical adolescent sample. BMC Psychiatry. 2013;13:272. https://doi.org/10.1186/1471-244x-13-2 72.

46. Gardvik KS, Rygg M, Torgersen T, Lydersen S, Indredavik MS. Psychiatric morbidity, somatic comorbidity and substance use in an adolescent psychiatric population at 3-year follow-up. Eur Child Adolesc Psychiatry. 2020. https://doi.org/10.1007/s00787-020-01602-8.

47. Kaufman J, Birmaher B, Brent D, Rao U, Flynn C, Moreci P, et al. Schedule for affective disorders and schizophrenia for school-age children-present and lifetime version (K-SADS-PL): initial reliability and validity data. J Am Acad Child Adolesc Psychiatry. 1997;36(7):980-8. https://doi.org/10.1097/00004 583-199707000-00021.

48. American Psychiatric Association. Diagnostic and statistical manual of mental disorders: DSM-IV. 4th ed. Text Revision ed. 2000. Washington (DC): American Psychiatric Association; 2000.

49. Miller CJ, Miller SR, Newcorn JH, Halperin JM. Personality characteristics associated with persistent ADHD in late adolescence. J Abnorm Child Psychol. 2008;36(2):165-73.

50. Lund LK, Vik T, Skranes J, Brubakk AM, Indredavik MS. Psychiatric morbidity in two low birth weight groups assessed by diagnostic interview in young adulthood. Acta Paediatr. 2011;100(4):598-604.

51. de Vet HC, Mokkink LB, Terwee CB, Hoekstra OS, Knol DL. Clinicians are right not to like Cohen's K. BMJ. 2013;346:f2125.

52. Shaffer D, Gould MS, Brasic J, Ambrosini P, Fisher P, Bird H, et al. A children's global assessment scale (CGAS). Arch Gen Psychiatry. 1983;40(11):1228-31.

53. Fagerland MW, Lydersen S, Laake P. Statistical analysis of contingency tables. Boca Raton: CRC Press/Taylor \& Francis Group; 2017.

54. Kessler RC, McLaughlin KA, Green JG, Gruber MJ, Sampson NA, Zaslavsky $\mathrm{AM}$, et al. Childhood adversities and adult psychopathology in the WHO world mental health surveys. Br J Psychiatry. 2010;197(5):378-85.

55. Copeland WE, Shanahan L, Hinesley J, Chan RF, Aberg KA, Fairbank JA, et al. Association of childhood trauma exposure with adult psychiatric disorders and functional outcomes. JAMA Netw Open. 2018;1(7):e184493.

56. Cecil CA, Viding E, Fearon P, Glaser D, McCrory EJ. Disentangling the mental health impact of childhood abuse and neglect. Child Abuse Negl. 2017;63: 106-19.

57. Evans E, Hawton K, Rodham K. Factors associated with suicidal phenomena in adolescents: a systematic review of population-based studies. Clin Psychol Rev. 2004;24(8):957-79.

58. Qin P, Agerbo E, Mortensen PB. Suicide risk in relation to socioeconomic, demographic, psychiatric, and familial factors: a national register-based study of all suicides in Denmark, 1981-1997. Am J Psychiatry. 2003;160(4): 765-72

59. Heiervang E, Stormark KM, Lundervold AJ, Heimann M, Goodman R, Posserud MB, et al. Psychiatric disorders in Norwegian 8- to 10-year-olds: an epidemiological survey of prevalence, risk factors, and service use. J Am Acad Child Adolesc Psychiatry. 2007:46(4):438-47. https://doi.org/10.1097/ chi.0b013e31803062bf.

\section{Publisher's Note}

Springer Nature remains neutral with regard to jurisdictional claims in published maps and institutional affiliations.

\section{Ready to submit your research? Choose BMC and benefit from:}

- fast, convenient online submission

- thorough peer review by experienced researchers in your field

- rapid publication on acceptance

- support for research data, including large and complex data types

- gold Open Access which fosters wider collaboration and increased citations

- maximum visibility for your research: over $100 \mathrm{M}$ website views per year

At BMC, research is always in progress.

Learn more biomedcentral.com/submissions 